\title{
Statistics of lowest droplets in two-dimensional Gaussian Ising spin glasses
}

\author{
M. Picco, ${ }^{1}$ F. Ritort, ${ }^{1,2}$ and M. Sales ${ }^{2}$ \\ ${ }^{1}$ LPTHE, Université Pierre et Marie Curie, Paris VI et Université Denis Diderot, Paris VII Boîte 126, Tour 16, $1^{e r}$ étage, \\ 4 place Jussieu, F-75252 Paris Cedex 05, France \\ ${ }^{2}$ Departament de Física Fonamental, Facultat de Física, Universitat de Barcelona, Diagonal 647, 08028 Barcelona, Spain
}

(Received 7 November 2002; published 30 May 2003)

\begin{abstract}
An approach to determine the value of the zero-temperature thermal exponent $\theta$ in spin glasses is presented. It consists in describing the energy level spectrum in spin glasses only in terms of the properties of the lowest energy droplets and the lowest droplet exponents (LDE's) $\lambda_{l}, \theta_{l}$ that describe the statistics of their sizes and gaps. We show how these LDE's yield the standard thermal exponent of droplet theory $\theta$ through the relation $\theta=\theta_{l}+d \lambda_{l}$. The present approach provides a new way to measure the thermal exponent $\theta$ without any assumption about the correct procedure to generate typical low-lying excitations as is commonly done in many perturbation methods including domain wall calculations. To illustrate the usefulness of the method we present a detailed investigation of the properties of the lowest energy droplets in two-dimensional Gaussian Ising spin glasses. By independent measurements of both LDE's and an aspect-ratio analysis, we find $\theta(2 d) \simeq$ $-0.46(1)<\theta_{\mathrm{DW}}(2 d) \simeq-0.287$ where $\theta_{\mathrm{DW}}$ is the thermal exponent obtained in domain-wall theory. We also discuss the origin of finite-volume corrections in the behavior of the LDE $\theta_{l}$ and relate them to the finitevolume corrections in the statistics of extreme values. Finally, we analyze some geometrical properties of the lowest energy droplets, finding results in agreement with those recently reported by Kawashima and Aoki [J. Phys. Soc. Jpn. 69, 169 (2000)]. All in all, we show that typical large-scale droplets are not probed by most of the present perturbation methods, since they probably do not have a compact structure as has been recently suggested. We speculate that a multifractal scenario could be at the roots of the reported discrepancies on the value of the thermal exponent $\theta$ in the two-dimensional Gaussian Ising spin glass.
\end{abstract}

DOI: 10.1103/PhysRevB.67.184421

PACS number(s): 75.10.Nr, 05.70.Jk, 75.40.Mg

\section{INTRODUCTION}

Despite three decades of work in the field of spin glasses, major issues related to their low-temperature behavior still remain unresolved. ${ }^{1}$ Although important achievements have been obtained in the understanding of mean-field theory ${ }^{2}$ the appropriate treatment beyond mean-field to include shortrange interactions is yet to be found. Due to the absence of a successful analytical approach to deal with this problem, the present state of our knowledge is often misguided by a nonaccurate, if not confusing, interpretation of the numerical data. This situation has generated a hot debate about the correct physical interpretation of the available numerical data. Leaving aside the long-standing controversy whether replica symmetry breaking is or not a good description of the spin-glass phase, ${ }^{3}$ there are still unresolved issues which are not as striking but show our ignorance about some fundamental questions.

One among these problems is the correct value of the thermal exponent in two-dimensional (2D) Gaussian Ising spin glasses (GISG's). This question has received attention from time to time during the last two decades, but not enough to settle it definitively and explain the origin of some of the reported discrepancies. The study of the low- $T$ properties of the 2D GISG's starts with the work by McMillan, who proposed ${ }^{4}$ that thermal properties in spin glasses are determined by the scaling behavior of the typical largest excitations (commonly referred to as droplets) present in the system. This idea has been further elaborated and extended to deal with equilibrium and dynamical properties of spin glasses in a scenario nowadays referred to as droplet model. ${ }^{5}$
The low- $T$ behavior in spin glasses is determined by a spectrum of large scale gapless droplets with typical length $L$ and energy cost $E \sim L^{\theta}, \theta$ being the thermal exponent. As these droplets correspond to flipping some domains of spins (assumed to be compact clusters), the energy cost of these excitations arises from the set of unsatisfied bonds on their surface. The striking low- $T$ behavior in spin glasses arises from multiple energy cancellations occurring at the surface of the droplet. These cancellations can be seen as the result of a competition between energy and entropy effects: as the droplet becomes progressively larger there are more available conformations for the surface to minimize the energy cost of the unsatisfied bonds. In the absence of cancellations one would expect $\theta=(d-1) / 2$. However, as these cancellations are very important, the inequality $\theta<(d-1) / 2$ holds and $\theta$ is by far less than the maximum value $(d-1) / 2$. The value of the thermal exponent $\theta$ characterizes the low- $T$ critical behavior as it is related to the correlation length exponent $\nu$ where $\xi \sim T^{-\nu}$ by the identity $\nu=-1 / \theta$. McMillan also used domain-wall renormalization group ideas to introduce a practical way to determine the leading energy cost of these low-lying large-scale excitations. ${ }^{6}$ The method consists in measuring the energy defect of a domain-wall spanning the whole system obtained by computing the change of the ground state energy when switching from periodic to antiperiodic boundary conditions in one direction. Several works have used McMillan's method to determine the value of $\theta$ in two and three dimensions. ${ }^{7,8}$ Hereafter, in order to keep the discussion as clear as possible, we will denote by $\theta_{\mathrm{DW}}$ the estimate of the exponent $\theta$ obtained by domain-wall calculations. The initial value for $\theta_{\mathrm{DW}}$ reported by McMillan is 
$\theta_{\mathrm{DW}}=-0.281(5)$ for pretty modest lattice sizes $L=3-8$. Recent numerical results with much more powerful algorithms have reached sizes $L \simeq 500$ and confirmed the initial result with much larger accuracy ${ }^{9-11} \theta_{\mathrm{DW}}=-0.287(4)$. These studies would definitively close the problem if it were not by the existence of other alternative estimates of the exponent $\theta$, largely consistent among them, which yield a quite different value $\theta \simeq-0.47(2)$. We will denote this estimate by $\theta_{\mathrm{TF}}$ as several of these methods use transfer matrix. ${ }^{12}$ However, a word of caution is necessary here as the Monte Carlo method and other approaches that are not based on transfer matrix methods report values compatible with that estimate. For instance, Kawashima and Aoki used another method to estimate the stiffness exponent. ${ }^{13}$ The idea is to generate a droplet inside a box of size $L \times L$ that includes a fixed central spin, with the following procedure. First, the ground state is found with a standard algorithms (we will denote it by the reference configuration). Afterward, the spins at the boundaries of the box are fixed and the central spin is forced to flip respect to the reference configuration. The droplet of minimum energy that includes the central reversed spin and does not touch the boundaries is computed. The spanning length of the droplets generated in this way allows us to define the fractal dimension of both the surface (or perimeter for the two-dimensional case) and the volume. It is found that these minimum energy droplets have a fractal volume dimension smaller than 2 and the thermal exponent is $\theta=-0.42(5)$ in agreement with results obtained from MC methods ${ }^{14}$ and heuristic optimization algorithms. ${ }^{15} \mathrm{~A}$ similar study of minimum energy clusters in the three-dimensional Edwards-Anderson model also reports evidence that $\theta_{\mathrm{DW}}$ is an upper bound to the actual value of the thermal exponent. ${ }^{16}$

The accuracy of previous estimates is poorer than the values obtained through the domain-wall method as they deal, in one way or another, with all possible excitations and not only with the calculation of ground state energies. More recently, another method has been used to estimate the value of $\theta$. It consists in perturbing the original Hamiltonian $\mathcal{H}_{0}$ with a term $\epsilon \mathcal{P}$, where $\mathcal{P}$ stands for the perturbation and $\epsilon$ for its intensity. For example, $\mathcal{P}$ can be the overlap between the actual configuration and the ground state of the original Hamiltonian $\mathcal{H}_{0}$. As $\epsilon$ varies the new ground state of the total Hamiltonian $\mathcal{H}=\mathcal{H}_{0}+\epsilon \mathcal{P}$ remains unchanged until a certain value $\epsilon=\epsilon_{c}$ is reached where a excited energy level of $\mathcal{H}_{0}$ becomes the new ground state of $\mathcal{H}$. The overlap between the old and the new ground states as well as the value of the shifting energy provoked by the perturbation links its energy cost $E$ with its size providing another way to estimate $\theta$. We will denote by $\theta_{P}$ the estimate obtained in this way. This method has been recently used in the $2 \mathrm{D}$ GISG by Hartmann and Young ${ }^{17}$ reporting the value $\theta_{P} \approx$ -0.31 . Although slightly more negative than $\theta_{\mathrm{DW}}, \theta_{\mathrm{DW}}$ and $\theta_{P}$ appear to be statistically compatible. Yet more accurate estimates are needed to confirm whether $\theta_{P}=\theta_{\mathrm{DW}}$.

This last method and the domain-wall method have in common the same feature, i.e., they perturb the original Hamiltonian in one way or another to probe the characteristic energy of excitations that are supposed to be the typical ones that determine the low- $T$ thermodynamic properties. In fact, the estimate $\theta_{\mathrm{DW}}$ can be considered as a particular example of $\theta_{P}$, where the perturbation consists in reversing all the bonds in one of the surfaces of the box. This raises the important question whether the different estimates of $\theta_{P}$, obtained by considering different class of perturbations, are different. The question is rather subtle as there are numerical indications that indeed this could be the case. For instance, ${ }^{8}$ measurements of $\theta_{P}$ where the perturbation is a uniform magnetic field yield a value $\theta_{P}=-0.48(1)$ compatible with the other competing set of values $\theta_{\mathrm{TF}}$.

How is it that the value of the exponent $\theta_{P}$ could depend on the type of perturbation? This is a very difficult question to answer, as our present knowledge is inadequate. We can offer only speculative answers. Strong discrepancies among different types of perturbations could arise if a multifractal scenario governs the statistics of excitations in spin glasses. By definition, in all perturbation methods the probed large scale droplets are those which minimize the energy cost but constrained to maximize the value of the perturbation for the selected droplets. Therefore, among all possible large-scale low-lying droplets the perturbation method selectively probes those that maximally overlap with the perturbation. A dependence of the value $\theta_{P}$ on a given class of perturbations could arise if the perturbation selectively probes one or another topological property of the droplet. This rather awkward multifractal scenario is not new in the field of disordered systems. Multifractality is known to be present in the localization problem in the strongly disordered regime. A multifractal scenario would imply the existence of different critical exponents at $T=0$, depending on the type of perturbation applied. On the other hand, the fact that the value estimated for $\theta_{P}$ when the perturbation is a uniform magnetic field appears to be consistent with the value $\theta_{\mathrm{TF}}$, suggests that maybe some types of perturbation can probe the relevant excitations while others may not. These good observables, which probe the typical excitations, could be called neutral observables in the same spirit as this term has been coined to describe observable dependences of the fluctuationdissipation ratio (i.e., the effective temperature) in glassy systems. Concomitantly, this "perturbation class dependence" issue is presently also debated in the different (but related to a certain degree) field of glassy dynamics.

If the hypothetical multi-fractal scenario holds, then we must face the question about what is the correct procedure to determine the thermal exponent $\theta$. As $\theta$ determines the free energy cost of droplets, the natural answer is that $\theta$ is given by the lowest value among all possible estimates

$$
\theta=\min _{\mathcal{P}}\left\{\theta_{\mathcal{P}}\right\} .
$$

With the present available data this relation suggests that the estimate $\theta_{\mathrm{TF}}$ is the correct value of the thermal exponent and that $\theta_{\mathrm{DW}}$ as well as many other estimates $\theta_{P}$ are only upper bounds to the true value.

The question we want to address in this paper is the following. Is it possible to devise a method that is alternative to current perturbation methods, in which excitations are not selectively probed by the perturbation, but selected only ac- 
cording to the correct balance between energy and entropy? The main purpose of this paper is to show that the analysis of the statistics of the first or lowest excitations gives a positive answer to this question. As we will see, the method we propose in this paper yields a consistent estimate of $\theta$ compatible with the value $\theta_{\mathrm{TF}}$, therefore supports the result that $\theta_{\mathrm{DW}}$ and many other $\theta_{P}$ are only upper bounds to the actual value of $\theta$. A preliminary account of these results has already appeared in Ref. 18.

The paper is divided as follows. Section II describes the basis of the lowest droplet approach and introduces the lowest droplet exponents. Section III shows the results obtained in the 2D GISG. Section IV analyzes a method to extract the value of the thermal exponent $\theta$. Section $\mathrm{V}$ presents a more powerful method to extract the value of the lowest droplet exponents based on an aspect-ratio analysis. Section VI discusses the origin of the finite-volume corrections to the value of the lowest droplet exponent $\theta_{l}$ as a problem of corrections in the statistics of extreme values. Section VII analyzes some topological properties of the lowest droplets. Finally, Sec. VIII presents the conclusions. There are also two technical appendixes. Appendix A presents the heuristic argument that $\theta_{l}=-d$ for Gaussian spin glasses, and Appendix B explains the transfer matrix method we used to obtain the lowest droplets.

\section{BASIS OF THE LOWEST DROPLET APPROACH}

The purpose of this work is to show an alternative approach to determine the low- $T$ behavior of spin glasses by studying the size and energy spectrum of the lowest excitations by introducing two exponents $\left(\lambda_{l}\right.$ and $\left.\theta_{l}\right)$ needed to fully characterize the zero-temperature fixed point. All through the paper we will denote these exponents as lowest energy droplet exponents or lowest droplet exponents in short, and that we will abbreviate as LDE's. The exponent $\lambda_{l}$ is the most important one and describes the probability of finding a large-scale lowest excitation spanning the whole system, while the exponent $\theta_{l}$ describes the system-size dependence of the average energy cost of these lowest excitations.

The underlying theoretical background of the approach is the following. To investigate the leading low-temperature behavior in spin glasses let us consider expectation values for moments of the order parameter by keeping only the ground state and the first or lowest excitation. This approach was introduced in Ref. 19 and can be shown to capture the lowtemperature behavior at the leading order. The method that investigates the low- $T$ properties based on a restricted analysis of the spectrum to the absolute lowest excitations has also been used for the study of the localized phase in the disordered Anderson model. ${ }^{20}$ The present paper can be seen as the applicability of these ideas to the spin-glass case. At the end of the paper (see Sec. VIII) we will give reasons supporting the validity of our approach.

To generate the spectrum of lowest excitations we consider the following procedure. Let us consider a set of $\mathcal{N}_{s}$ samples and for each of them we determine both the configurations of the ground state and the lowest excitation. For a spin model the lowest excitation has $v$ spins overturned with respect to the ground state (so the overlap between the ground and that excited state is $q=1-2 v / V, V$ being the volume of the system) and with energy cost or gap $E$. It can be easily proved that the lowest excitation must be a connected cluster which we will generically call the lowest droplet. If $v_{s}$ and $E(s)$ denote the volume and excitation energy of the lowest droplet for sample $s$, in the limit where $\mathcal{N}_{s}$ is sent to infinity, we can define the following joint probability distribution:

$$
P(v, E)=\frac{1}{\mathcal{N}_{s}} \sum_{s=1}^{\mathcal{N}_{s}} \delta\left(v-v_{s}\right) \delta[E-E(s)] .
$$

Using the Bayes theorem, this joint probability distribution can be written as $P(v, E)=g_{v} \hat{P}_{v}(E)$, where

$$
\sum_{v=1}^{V / 2} g_{v}=1, \quad \int_{0}^{\infty} d E \hat{P}_{v}(E)=1 \quad \forall v
$$

$g_{v}$ is the probability to find a sample such that its lowest droplet has volume $v$ and $\hat{P}_{v}(E)$ is the conditioned probability for that droplet to have a gap equal to $E$. In what follows, we separately discuss the scaling behavior of both distributions $g_{v}, \hat{P}_{v}(E)$.

Before continuing, and for sake of clarity, let us make an important digression about nomenclature. There are two volumes involved in the problem: the volume $v$ of the lowest excitation and the volume $V$ of the lattice. If not stated otherwise we will refer to the volume $v$ as the size of the excitation while volume will generally refer to the lattice volume $V$. Thus, when we speak about finite-size excitations we usually refer to excitations with $v$ finite, and finite-volume corrections (which we will sometimes abbreviate as FVC) will refer to the corrections affecting the distribution (2) due to the finite volume $V$ of the lattice.

\section{A. The lowest droplet exponent $\boldsymbol{\lambda}_{l}$}

The simplest scenario for the size distribution of the lowest droplets is that all sizes occur with uniform probability. The normalization condition (3) imposes $g_{v} \sim 1 / V$. This situation is encountered in the $1 \mathrm{D}$ GISG ${ }^{5,19}$ with both free and periodic boundary conditions. However, in the most general situation, this does not hold and low energy droplets are found with a probability that depends on their size $v$. The simplest and most general way to incorporate such a dependence is to assume an ansatz solution for $g_{v}$ that factorizes into a power law $A / V^{\lambda_{l}+1}$ with $\lambda_{l}>0$ and a coefficient $A$ $\equiv G(q)$ which depends only on the overlap $q$ between the ground state and the lowest droplet

$$
g_{v}=\frac{G(q)}{V^{\lambda_{l}+1}} .
$$

The behavior of $G(q)$ can be guessed in both limits $q \rightarrow 1$ (the case $q \rightarrow-1$ is equivalent in models with timereversal symmetry which are those we are considering here) and $q \rightarrow 0$ 


$$
\begin{gathered}
G(q \rightarrow 0) \rightarrow \text { const, } \\
G(q \rightarrow 1) \rightarrow \frac{1}{(1-q)^{\lambda_{l}+1}} .
\end{gathered}
$$

The first relation describes the scaling behavior for the number of droplets whose size scales with the total volume of the system. As these can only depend on the volume $V, G(0)$ must converge to a constant. The second relation is consequence of the fact that the number of droplets with finite size $v$ cannot depend on $V$ in the large $V$ limit as these are not affected by the boundaries. On the other hand, the distribution of finite size droplets $g_{v}$ is self-similar as can be seen by inserting Eq. (6) in Eq. (4) and using the relation $q=1$ $-2 v / V$. This yields $g_{v} \sim 1 / v^{\lambda_{l}+1}$, the same relation as for the large scale limit (5), where $g_{V} \sim 1 / V^{\lambda_{l}+1}$. A simple expression that interpolates both limits is given by

$$
G(q)=\left(A+\frac{B}{(1-q)^{\lambda_{l}+1}}\right) \text {. }
$$

Note however that, despite its simplicity, expression (7) is only an interpolation and the most we can say about $G(q)$ concerns its asymptotic behaviors (5),(6).

The ansatz (4), applied only to large-scale excitations, was proposed in Ref. 19. Note that although $g_{v}$ is defined for discrete volumes, in the limit $V \gg 1$, the values of $q$ for consecutive droplet sizes $v \rightarrow v+1$ become equally spaced by $\Delta q=2 / V$. Therefore, in the limit, $V \gg 1$, the function $g(q)$ $=(V / 2) g_{v}$ becomes a continuous function if expressed in terms of the variable $q$ instead of the integer variable $v$,

$$
g(q)=\frac{1}{2 V^{\lambda_{l}}} G(q)
$$

A word of caution is in order. Although Eq. (4) diverges for $q=1$, leading apparently to a violation of the normalization condition (3) for $g_{v}$, it must be emphasized that no excitation has $q=1$ so there is a maximum cutoff value $q^{*}=1$ $-2 / V$ corresponding to one-spin excitations. For instance, if we insert Eq. (8) into the normalization condition for $g(q)$ we get in the large $V$ limit,

$$
\int_{0}^{q^{*}=1-2 / V} g(q) d q=1 \rightarrow \frac{A-B / \lambda_{l}}{2 V^{\lambda_{l}}}+\frac{B}{2^{\lambda_{l}+1} \lambda_{l}}=1,
$$

implying $\lambda_{l} \geqslant 0$ as expected since otherwise the normalization would not be possible in the large- $V$ limit. The divergent term $(q \rightarrow 1)$ in Eq. (8) shows that for $\lambda_{l}>0$ one-spin excitations are the most numerous among the whole spectrum of sizes. In fact, from Eq. (4), $g(1) \simeq O(1) \gg g(V / 2) \simeq 1 / V^{\lambda+1}$, so the majority of excitations have a finite size. However, the average excitation size

$$
\bar{v}=\sum_{v=1}^{V} v g_{v} \rightarrow V \rightarrow \infty V^{1-\lambda_{l}}
$$

diverges in the $V \rightarrow \infty$ limit and differs from the typical excitation volume $v_{\text {typ }} \sim O(1)$. Relation (10) provides a way to measure the exponent $\lambda_{l}$ alternative to the use of the scaling behavior (4).

\section{B. The lowest droplet exponent $\theta_{l}$}

The analysis of the gap distribution $\hat{P}_{v}(E)$ goes along the same lines as we did for the distribution $g_{v}$, but with one important difference. As the gap $E$ describes the lowest among all possible excitation energies, it has to scale in the same way for all droplet sizes independently on their size (and, in particular, whether these are finite-size or large scale droplets). This statement refers to a scenario which hereafter we will call the random energy-size droplet (RESD) scenario to specifically indicate that the distribution of the lowest energies of droplets is independent of their size. Mathematically it can be expressed as

$$
\hat{P}_{v}(E)=\hat{P}(E), \quad \forall v .
$$

In addition, we follow the standard droplet model and assume that the spectrum is gapless and defined by an exponent $\theta_{l}$ which describes the characteristic energy of the lowest droplets whatever their size or overlap $q$ with the ground state. If the scaling function $\hat{P}_{v}(E)$ is independent of $v$ it follows immediately that the non-conditioned or sizeaveraged gap probability distribution

$$
P(E)=\sum_{v \geqslant 1} g_{v} \hat{P}_{v}(E)=\sum_{v \geqslant 1} g_{v} \hat{P}(E)=\hat{P}(E)
$$

where we used Eq. (11) and the normalization condition (3) for $g_{v}$. From now on, if not stated otherwise, we will always refer to the size-averaged probability distribution $P(E)$ with the clear understanding that it coincides with any of the conditioned distributions $\hat{P}_{v}(E)$. As the spectrum of lowest excitations is gapless, the normalized distribution $P(E)$ has the following scaling behavior:

$$
P(E)=\frac{1}{L^{\theta_{l}}} \mathcal{P}\left(\frac{E}{L^{\theta_{l}}}\right)
$$

We stress that the exponent $\theta_{l}$ is completely different from the standard thermal exponent (see next section) as they describe totally different excitations. The thermal exponent $\theta$ describes the energy-length relation for droplets typically excited at finite temperatures while the lowest energy exponent $\theta_{l}$ describes the droplets that are separated by the smallest gap, respectively, to the ground state, so that, in general, $\theta_{l} \leqslant \theta$.

We will argue below in Sec. II C that $\theta_{l}=-d$ for a generic class of spin-glass systems with coupling distributions with finite weight at zero gap. In addition, this relation will provide an alternative interpretation of the lower critical dimension in terms of the exponent $\lambda_{l}$ introduced in Sec. II A describing the properties of the spectrum of sizes of the lowest droplets. 


\section{The standard thermal exponent $\theta$}

Now we want to show how the exponents $\lambda_{l}$ and $\theta_{l}$ combine to give the usual scaling exponent $\theta$ describing the energy cost of typical thermal excitations in droplet theory. There are several ways to show this result. For simplicity, here we exemplify this relation by analyzing the low- $T$ behavior of the second moment of the spin-glass order parameter at the order linear in $T$ by keeping only the first excitation. If $q_{\{\sigma, \tau\}}=(1 / V) \sum_{i} \sigma_{i} \tau_{i}$ denotes the overlap between two replicas (i.e., configurations of different systems with the same realization of quenched disorder), then the expectation value $\overline{\left\langle q^{2}\right\rangle}$ can be written as ${ }^{19}$

$$
\overline{\left\langle q^{2}\right\rangle}=1-\frac{2}{V^{2}} \sum_{v} \int_{0}^{\infty} d E P(v, E) v(V-v) \operatorname{sech}^{2}\left(\frac{E}{2 T}\right),
$$

where $P(v, E)$ is given by Eq. (2). A low-temperature expansion of Eq. (14) (Refs. 19,18) up to linear order in $T$ yields

$$
\overline{\left\langle q^{2}\right\rangle}=1-\frac{4 T}{V^{2}} \sum_{v=1}^{V} g_{v} \hat{P}_{v}(0) v(V-v)
$$

which shows that the leading behavior is determined by both $g_{v}$ and the density of states at zero gap $\hat{P}_{v}(0)$. In the standard droplet model, it is generally assumed that typical low energy droplets have an average size $\bar{v}=\Sigma_{v} v g_{v} \sim V$ of the order of the system size (such as those generated by DW perturbation) and finite weight at zero gap $\hat{P}_{V}(0) \sim 1 / L^{\theta}$ where $\theta$ is the thermal exponent. In principle, a single exponent $\theta$ describes the scaling behavior of typical large-scale droplets with volume $v \propto V$ and determines the zerotemperature critical behavior. As these large-scale droplets are typical they occur with finite (therefore independent of $V)$ probability $g_{V} \sim O(1)$ while small scale droplets are simply irrelevant $g_{v \sim O(1)} \sim 0$. This yields

$$
\overline{\left\langle q^{2}\right\rangle}=1-c \frac{T}{L^{\theta}}
$$

where $c$ is a nonuniversal stiffness constant related to the particular model. One of the most relevant results from the ansatz (4) is that both small and large scale excitations contribute to low-temperature properties. In general, let us consider any expression [such as Eq. (15)] involving a sum over all possible volume excitations. Restricting the sum to the large-scale droplets $(v / V$ finite) the net contribution to such sum is proportional to $V g_{V} \hat{P}_{V}(0) \propto L^{-\theta_{l}-d \lambda_{l}} \mathcal{P}(0)$ [where $\mathcal{P}$ is the scaling function appearing in Eq. (13)]. Coming back to Eq. (15) and using Eqs. (4) and (13), we note that both small and large-scale excitations yield a contribution to Eq. (15) of the same order and given by

$$
\overline{\left\langle q^{2}\right\rangle}=1-c_{l} \frac{T}{L^{\theta_{l}+d \lambda_{l}}},
$$

where $c_{l}$ is another constant [different from the constant $c$ appearing in Eq. (16)]. Identifying both relations (16) and (17) we obtain the general relation

$$
\theta=\theta_{l}+d \lambda_{l}
$$

This relation shows how the value of $\theta$ can be computed from $\lambda_{l}$ and $\theta_{l}$. Through the study of a specific example, we will see later that the exponents $\theta_{l}$ and $\lambda_{l}$ have strong finitevolume corrections arising from the corrections present in the statistics of the extreme values. However, we will present alternative routes to overcome this dependence and provide an accurate estimate of $\theta$.

Now we come back to the aforementioned argument at the end of Sec. II B claiming that in the large-volume limit $\theta_{l}$ must converge to the value $-d$ in the case of coupling distributions with finite gap at zero coupling. The details of the argument are shown in Appendix A. The argument has two parts. First, it is proved that one-spin excitations provide an upper bound for the LDE $\theta_{l}$. Then it is argued that this upper bound holds also for any finite-size excitations (such as twospin clusters, three-spin clusters, and so on). We will see below how this result is supported by the numerical analysis of the data. Let us also note that this result, in a RESD scenario (see Sec. II B) can be linked to the linear dependence of the specific heat at low temperatures, a result widely accepted, but that has been revisited recently in Ref. 21 to show that it has strong FVC due to the systematic FVC present in the value of $\theta_{l}$. Inserting $\theta_{l}=-d$, Eq. (18) becomes

$$
\theta=d\left(\lambda_{l}-1\right)
$$

This relation provides a way to distinguish the lower critical dimension $d_{\mathrm{LCD}}$ in terms of the average size distribution of the lowest droplets. According to Eq. (10) the relation $\lambda_{l}\left(d_{\mathrm{LCD}}\right)=1$ distinguishes a regime where the average size of the lowest droplet grows with the volume of the system to a regime where the average size of the lowest droplet is finite,

$$
\begin{gathered}
d<d_{\mathrm{LCD}}: \lim _{V \rightarrow \infty} \bar{v}(V)=\infty, \quad \lambda_{l}<1, \theta<0, \\
d>d_{\mathrm{LCD}}: \lim _{V \rightarrow \infty} \bar{v}(V)=O(1), \quad \lambda_{l}>1, \theta>0 .
\end{gathered}
$$

The marginal case $\lambda_{l}=1, \theta=0$ is specially interesting as the average size $\bar{v}$ could be finite or diverge with the size but slower than a power law. This scenario corresponds to the mean-field behavior as replica symmetry is broken in both the standard RSB (Ref. 3) or in the trivial-nontrivial ${ }^{22}$ (TNT) scenarios. Therefore, the study of the size spectrum of the lowest excitations in spin glasses can be very useful to find out the correct value of the thermal exponent in models without a finite- $T$ transition (such as the 2D GISG) as well as establishing the correct low- $T$ scenario in models with a finite- $T$ transition. In the next section we apply all these ideas to evaluate the thermal exponent for the 2D GISG's. 

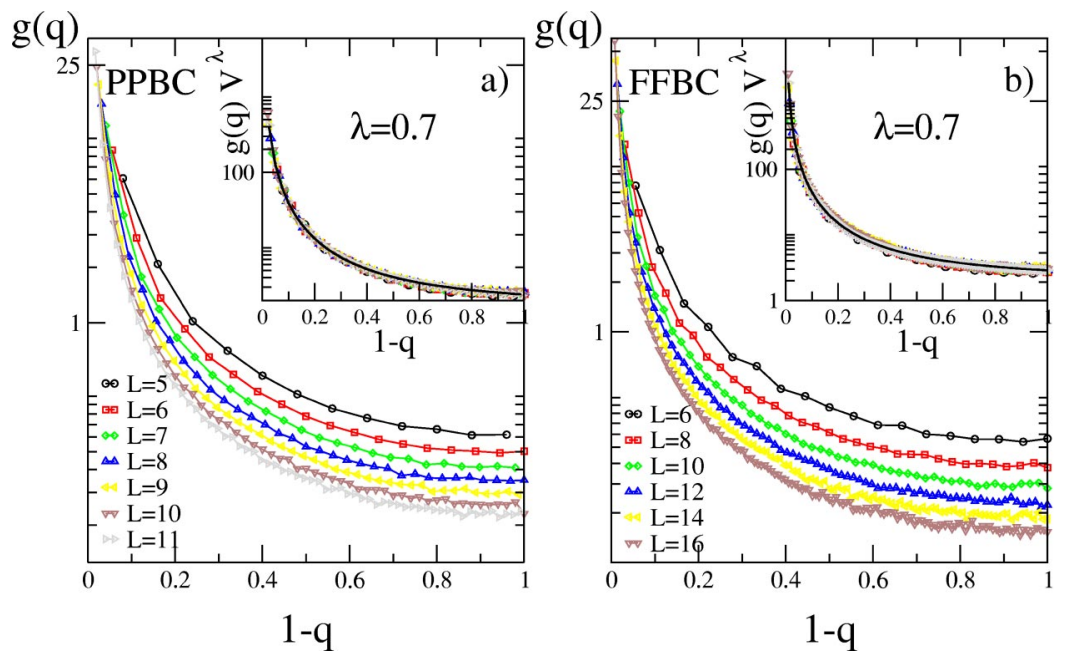

FIG. 1. $g(q)$ versus $1-q$ for the PP (left panel) and FF case (right panel) for different lattice sizes $L=5-11(\mathrm{PP})$ and $L=6-16(\mathrm{FF})$ from top to bottom. In both insets we plot the scaling function $g(q) V^{\lambda}$ versus $1-q$ with $\lambda=0.7$.

\section{STATISTICS OF THE LOWEST ENERGY DROPLETS IN THE 2D GISG}

Several numerical works have recently searched for lowlying excitations in spin glasses using heuristic algorithms. ${ }^{23}$ But, to our knowledge, no study has ever presented exact results about the statistics of lowest excitations. We have exactly computed ground states and lowest excitations in two-dimensional Gaussian spin glasses defined by

$$
\mathcal{H}=-\sum_{i<j} J_{i j} \sigma_{i} \sigma_{j}
$$

where the $\sigma_{i}$ are the spins $( \pm 1)$ and the $J_{i j}$ are quenched random variables extracted from a Gaussian distribution of zero mean and unit variance. These have been computed by using a transfer matrix method working in the spin basis. Representing each spins state by a weight and a graduation in the energy we can build explicitly the ground state by keeping the largest energy and, by subsequent iteration, the first excitation and so on (see Appendix B for the details on how we compute these quantities). The continuous values for the couplings assures that there is no accidental degeneracy in the system (apart from the trivial time-reversal symmetry $\sigma \rightarrow-\sigma)$. Calculations have been done in systems with free boundary conditions in both directions (FF), periodic boundary conditions in both directions (PP), and free boundary conditions in one direction but periodic in the other (FP). In all cases we find the same qualitative and quantitative results indicating that we are seeing the correct critical behavior.

We have found ground states and lowest droplets for systems ranging from $L=4$ up to $L=11$ for PP and up to $L$ $=16$ for FP and FF. The number of samples is very large, typically $10^{6}$ for all sizes. The large number of samples assures us that many samples have large-scale droplets as first excitations. This provides us with good statistics to properly analyze the sector of large-scale excitations. The large number of samples requires a big amount of computational time so that calculations were done in a PC cluster during several months. For each sample we have evaluated the volume of the excitation $v$ (and hence the overlap $q=1-2 v / V$ be- tween the ground state and the first excitation) and the gap $E$. From these quantities we can construct the $g_{v}$ and the $\hat{P}_{v}(E)$.

In Fig. 1 we show $g(q)=(V / 2) g_{v}$ as function of $q$ for different sizes in the PP and FF cases. We can clearly see that there are excitations of all possible sizes but, as discussed in the paragraph following Eq. (9), the typical ones which dominate by far are single spin excitations. To have a rough idea of the number of rare samples giving large scale excitations let us say that nearly half of the total number of samples have one-spin lowest excitations, whereas less than $10 \%$ of the samples have lowest excitations with overlap $q$ in the range $0-0.5$. This disparity increases systematically with size. For the lattice sizes explored the typical number of large-scale droplets is in the range $10^{4}-10^{5}$ which is, indeed, quite good to have a good sampling of the sector corresponding to large scale excitations. A detailed analysis of the shape of $g_{v}$ reveals that it has a flat tail for large-scale excitations and a power-law divergence for finite-size excitations. The $g_{v}$ can be excellently fitted by the interpolating formula [Eqs. (7),(8)],

$$
g(q)=\frac{2}{V^{\lambda_{l}}}\left(A+\frac{B}{(1-q)^{\lambda_{l}+1}}\right) .
$$

As shown in the insets of Fig. 1 a good collapse of the scaling function is obtained with the effective exponent $\lambda_{l}^{\text {eff }}$ $\simeq 0.7$ for both PP and FF cases. We also plot the line resulting from the fit of Eq. (23) with numerical data with the following values for $A$ and $B$ : PPBC: $A=1.55(3)$ and $B$ $=0.777(3)$; FFBC: $A=2.02(3)$ and $B=0.85(1)$. Note that the fit is excellent and is hardly distinguishable from the points. The value of $\lambda_{l}$ is compatible with the one obtained by fitting the average size with the expression (10) with the addition of a constant term to account for the small- $V$ behavior $\bar{v}=C_{1}+C_{2} V^{1-\lambda}$. The same exponent $\lambda_{l}$ can be estimated by measuring the ratio $g(V / 2) / g(1) \sim D_{1}$ $+D_{2} V^{-1-\lambda}$. In both cases we get an effective exponent $\lambda_{l}^{\mathrm{eff}}=0.70(5)$ as best fitting value.

However, these different estimates of $\lambda_{l}$ are strongly affected by finite-volume corrections (FVC's). To evidence 


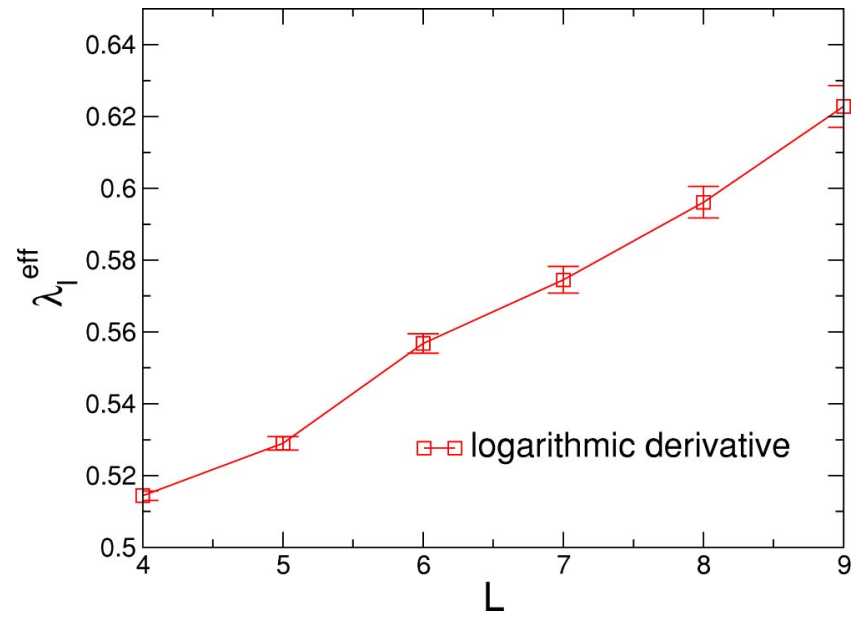

FIG. 2. Effective lowest droplet exponent $\lambda_{l}^{\text {eff }}$ versus $L$ for the PP case, computed using logarithmic derivatives.

them we have estimated an effective $L$ dependent $\lambda_{l}^{\text {eff }}(L)$ exponent by relating the average excitation size at consecutive sizes and using relation (10)

$$
\lambda_{l}^{\mathrm{eff}}(L)=1-\frac{1}{d} \frac{\ln \left(\frac{\bar{v}(L+1)}{\bar{v}(L)}\right)}{\ln \left(\frac{L+1}{L}\right)} .
$$

In Fig. 2 we show $\lambda_{l}^{\text {eff }}(L)$ in the range $L=4-11$ for the PP case. As we can appreciate there is a systematic increase of the effective exponent as we go to large volume sizes without any tendency to saturate. This proves that FVC in our measurements are still big and the estimate $\lambda_{l}^{\text {eff }}$ used to collapse the data in Fig. 1 is still far from the asymptotic exact value.

After having discussed the $g_{v}$ we jump now to discuss the scaling behavior of the energy gap distribution $\hat{P}_{v}(E)$ and its average $P(E)$. In Fig. 3 we show $P(E)$ [main figure and inset (a)] and $\hat{P}_{v}(E)$ [inset (b)] for the PP case. Similar results are obtained for the FF and FP cases. Quite remarkably, as was already anticipated in Eq. (11), the RESD scenario holds as the distribution $\hat{P}_{v}(E)$ does not depend on the size $v$ of the excitation [see inset (b) in Fig. 3], hence both large and finite-size excitations are described by the same gap distribution.

In the main figure we can see how the width of distribution $P(E)$ progressively shrinks to 0 as $L$ increases. Moreover, the $P(E)$ has an exponential shape. This is shown in the inset (a) of Fig. 3 where we plot $P(E)$ in log-normal scale. Nonetheless, a detailed examination of the tails of $P(E)$ reveals some deviations from linearity. In Sec. VI we discuss the origin of these deviations. We anticipate, though, that they are consequence of the strong $\mathrm{FVC}$ in the range of sizes investigated. In that inset we also verify the scaling ansatz (13) by showing the best data collapse for $P(E)$ obtained with an effective exponent $\theta_{l}^{\text {eff }} \simeq-1.7(1)$. This is very far from the expected value $\theta_{l}=-2$ discussed in the

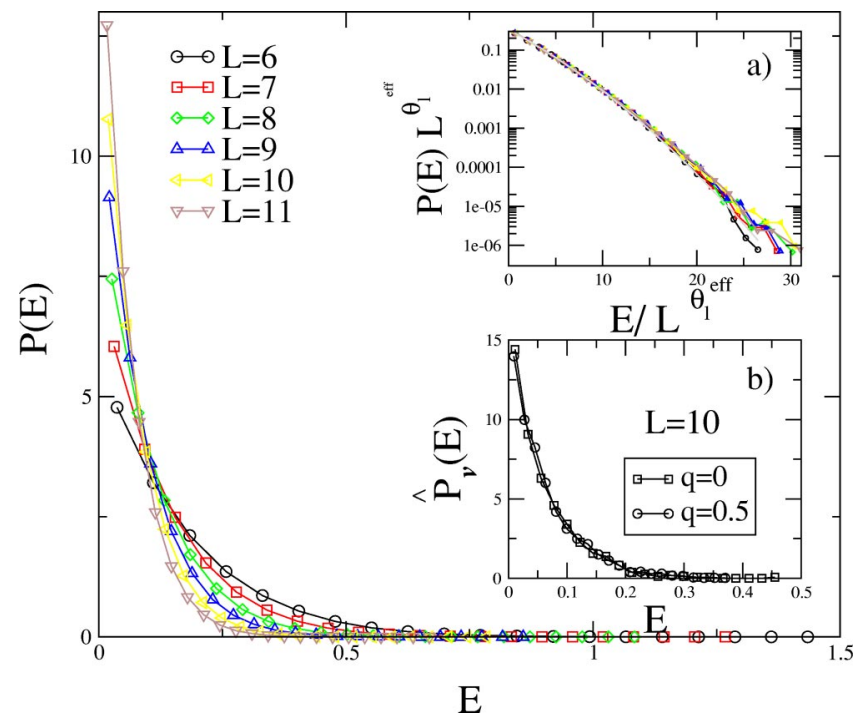

FIG. 3. Gap distribution $P(E)$ versus $E$ for different lattice sizes in the PP case. In inset (a) scaling obtained from the ansatz (13) with $\theta_{l}^{\text {eff }}=-1.7(1)$. In inset (b) we show the $\hat{P}_{v}(E)$ for different excitation sizes $(q=0.5, q=0)$ for a lattice size $L=10$. Note that the distribution is independent of the size of the excitation.

preceding Sec. II C and in the Appendix A. A calculation of the moments of $P(E)$ (13) for different values of $L$ shows that there are also strong sub-dominant corrections to the leading scaling (13) that result in corrections as large as the ones affecting the exponent $\lambda_{l}$.

Again, to manifest the magnitude of FVC in $\theta_{l}$ we have evaluated $\bar{E}(L)$, the first moment of $\hat{P}(E)$, obtained by averaging the lowest gap over all possible droplet sizes for different lattice sizes in the range $L=4-11$. We have estimated an effective $L$-dependent exponent by means of the following expression:

$$
\theta_{l}^{\mathrm{eff}}(L)=\frac{\ln \left(\frac{\bar{E}(L+1)}{\bar{E}(L)}\right)}{\ln \left(\frac{L+1}{L}\right)} .
$$

The results are shown in Fig. 4 for the PP case. Again, as for $\lambda_{l}^{\text {eff }}$ (see Fig. 2), we observe that the estimated value for $\theta_{l}^{\text {eff }}$ systematically changes with size showing that, for the sizes we have explored, we are still far from the asymptotic regime.

We can summarize the results of this section saying that both lowest droplet exponents (LDE's) $\lambda_{l}$ and $\theta_{l}$ display strong systematic finite-volume corrections (FVC). In principle, without further elaboration, it is difficult to give an accurate estimate for the thermal exponent $\theta$ using Eq. (18). An alternative estimate for the exponent $\theta$ could be defined from the analysis of the fraction of large-scale excitations with $q \leqslant 1 / 2, f(q \leqslant 1 / 2),{ }^{24}$ which is given by

$$
f(q \leqslant 1 / 2) \sim V g(0) \sim 1 / V^{\lambda_{l}-1} \sim L^{d\left(\lambda_{l}-1\right)} \sim 1 / L^{\theta},
$$




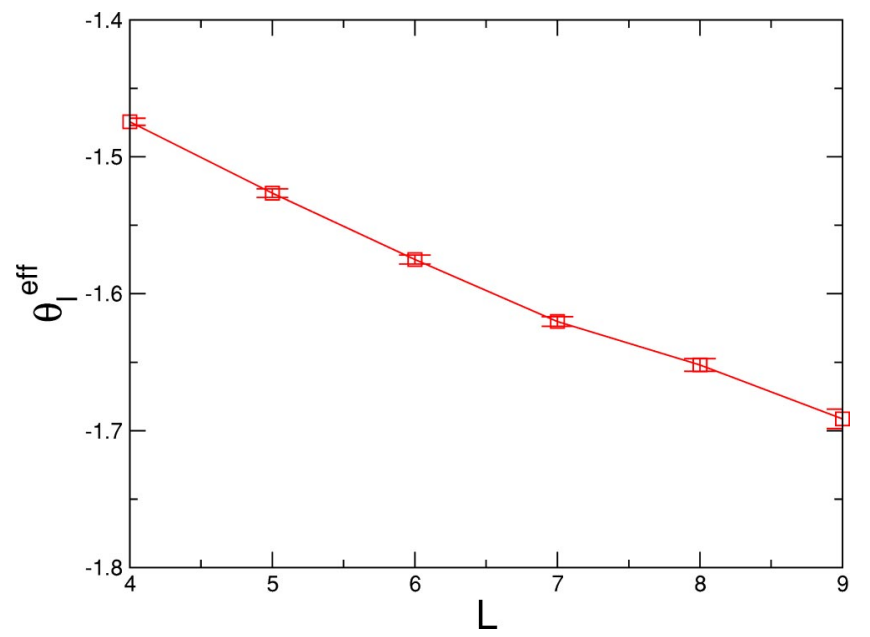

FIG. 4. Effective droplet exponent $\theta_{l}^{\text {eff }}$ versus $L$ for the PPBC case, computed using logarithmic derivatives (see text).

where we have used $\theta_{l}=-d$ (19). Although Eq. (26) yields estimates for $\theta$, again these are affected by strong finitevolume corrections. In the range of sizes studied in this paper, and using Eq. (26) we get $\theta \simeq-0.6$ quite far from the asymptotic value reported later in Secs. IV and V. How can we go further and estimate $\theta$ in a safer way? In the next two sections we shall answer this question.

\section{A GOOD ESTIMATE OF THE LOWEST DROPLET EXPONENTS}

An interesting aspect of the effective $L$-dependent exponents shown in Figs. 2 and 4 is that, while their FVC are large, their corrections are of opposite sign. While $\lambda_{l}^{\text {eff }}(L)$ increases with $L, \theta_{l}^{\text {eff }}(L)$ decreases. As they have to be added to get $\theta$ according to the relation (18) their finite-volume corrections cancel out to a certain degree. If we combine the two estimates for the best data collapse given in the previous section $\left[\lambda_{l}^{\text {eff }}=0.70(5), \quad \theta_{l}^{\text {eff }} \simeq-1.7(1)\right]$ we obtain $\theta \simeq$ $-0.3(2)$, which is very close to the DW value in average. But since the error on $\theta$ is so large, this estimate is not very useful. A better route would be to use the two LDE's estimated from Eqs. (24),(25) and adding them according to Eq. (18)

$$
\theta^{\mathrm{eff}}(L)=\theta_{l}^{\mathrm{eff}}(L)+d \lambda_{l}^{\mathrm{eff}}(L) .
$$

In Fig. 5 (left panel) we show the value of $\theta$ obtained in this way. Note that the value of the thermal exponent $\theta$ has negligible FVC but relatively large statistical fluctuations with $L$.

A better, albeit related, way to estimate $\theta$ is the following. Instead of independently finding out $\lambda_{l}$ and $\theta_{l}$ we look for an estimator which depends on the appropriate combination of the two exponents $\theta=\theta_{l}+d \lambda_{l}$. The simplest quantity which satisfies this requirement is given by the combination

$$
A(L)=L \frac{\bar{E}(L)}{\bar{v}(L)} .
$$

Since $\bar{E}(L) \simeq L^{\theta_{l}}$ and $\bar{v}(L) \simeq L^{d\left(1-\lambda_{l}\right)}$, using Eq. (18) we obtain $A(L) \sim L^{\theta}$. To estimate the value of $\theta$ we follow two different routes: (1) We use Eq. (25) by replacing $\theta_{l}^{\text {eff }}(L)$ $\rightarrow \theta^{\text {eff }}(L)$ and $\bar{E}(L) \rightarrow A(L)$. By definition, this procedure gives exactly the estimate (27) shown in the left panel in Fig. 5. (2) A more stable estimate can be obtained from a fit of $A(L)$ versus $L$, with data in the range $\left[L, \ldots, L_{\max }=11\right]$ (for the PPBC case). This is shown in the left panel of Fig. 5 together with the previous estimate (27) and also in the right panel of Fig. 5 but there compared with the effective exponent $\theta_{\mathrm{DW}}$ obtained from domain-wall calculations. Our best value for $\theta$ is

$$
\theta=-0.46(1)
$$

This value is very close to the finite-temperature (Monte Carlo or transfer matrix) estimates $\theta_{\mathrm{TF}}=-0.48(1)$ (Ref. 15) but certainly smaller than the domain-wall value $\theta_{\mathrm{DW}}=$ -0.285 . $^{7,8}$ Our estimate for $\theta$ is compatible with the other possible value $\theta_{\mathrm{TF}}$ obtained by other methods as discussed in Sec. I but is certainly inconsistent with the value obtained with other methods with results closer to the DW estimate.

All these estimates strongly support the inequality $\theta$ $=\theta_{\mathrm{TF}}<\theta_{\mathrm{DW}}$. However, one cannot exclude a situation where the present tendency of the data gets modified and $\theta$ $\rightarrow \theta_{\mathrm{DW}}$ in the large- $L$ limit. ${ }^{25}$ We have already explained in Sec. II C that $\theta_{l}$ must converge to -2 in the large volume limit implying the relation (19). Introducing our estimate (29) in Eq. (19) we get

$$
\lambda_{l}=0.770(5) .
$$
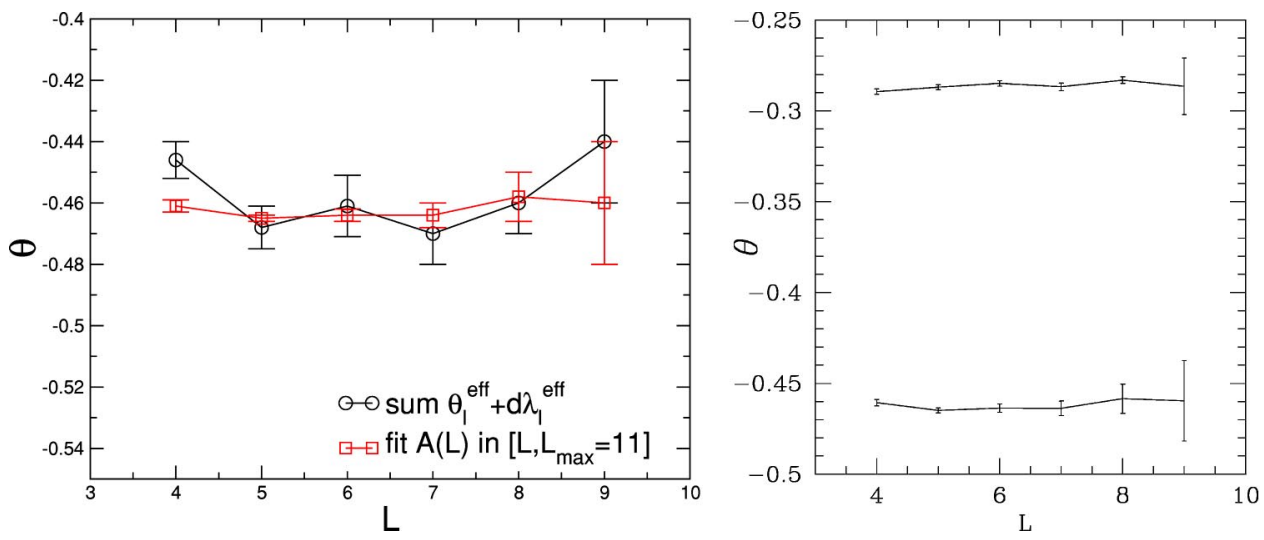

$184421-8$
FIG. 5. Exponent $\theta$ for the PPBC case. Left plot: $\theta$ exponent versus $L$ obtained from two methods. Method 1: using Eq. (27). Method 2: using the more stable estimate fitting (28) over a given range of $L$ values (see text). Right plot: Domain-wall exponent (top) and $\theta$ exponent (bottom) estimated by the second method as explained in the text and plotted as a function of $L$. 


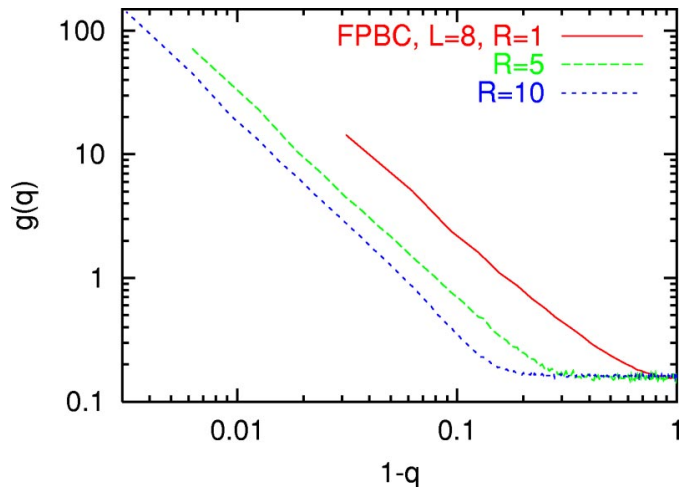

FIG. 6. $g(q)$ versus $1-q$ for the FPBC for $R=1,5$, and 10 and for $L=8$.

A convincing proof of the correctness of the values (29),(30) requires proving that the estimate $(24)$ converges to the value (30) when $L \rightarrow \infty$. In the next section we present an aspectratio analysis to evidence that the estimates (29),(30) are correct in the large $L$ limit.

\section{ASPECT-RATIO ANALYSIS OF THE LOWEST DROPLET EXPONENTS}

In this section, we present some additional data obtained via an aspect-ratio analysis (ARA). This analysis has been proved to be very useful to extract the value of the domainwall exponent $\theta_{\text {DW }}$ by generating domain walls in rectangular lattices $M \times L$ with different aspect ratios $M / L .^{10,11}$ It has been found that, in the limit of large aspect ratio, the value of $\theta_{\text {DW }}$ for Gaussian spin glasses is largely independent of the boundary conditions. We have seen in Sec. III that our measurements on squared lattices of size $L \times L$ mix small excitations with large ones so one does not have a clear-cut separation in the statistical distribution between the two different regimes $v \sim \mathcal{O}(1)$ and $v / V \sim \mathcal{O}(1)$. Our main motivation here is to show that, by investigating large aspect ratios, we can separate these two different scaling regimes. We made our measurements on systems of size $L \times M$, with $M=L R$ $\gg L$ where $R$ ranges from 1 up to 10 . We have investigated different types of boundary conditions: periodic boundary conditions in both directions (PPBC) and periodic boundary conditions in the $L$ direction with free boundary conditions in the $M$ direction (FPBC).

In Fig. 6, we display the data for $g(q)$ versus $1-q$ (8) for the FPBC case for $L=8$ and $R=1,5$, and 10 . One can clearly see that the behavior of the distribution $g(q)$ drastically changes as one increases $R$. Indeed, as we have already seen in Sec. III and in Eqs. (5), (6), and (8), for $R=1$ it is very difficult to separate the region of small excitations (a scaling region with $\left.g(q) \simeq 1 /(1-q)^{\lambda_{l}+1}\right)$ from the one of large excitations (a constant $q$-independent contribution $\left.g(q) \simeq 1 / V^{\lambda_{l}}\right)$. The main advantage of separating these two regions is that one can directly fit each of them. This yields two separate measurements of the $\operatorname{LDE} \lambda_{l}$ in addition to the estimate (10) obtained from the $L$ dependence of the average size of the excitations.

In Fig. 7, we show $g(q)$ versus $1-q$ for $R=10$ for vari-

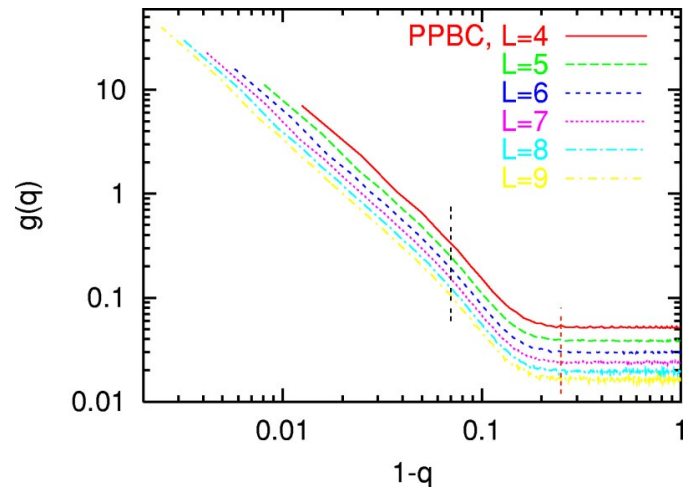

FIG. 7. $g(q)$ versus $1-q$ for the PPBC case for $R=10$.

ous linear sizes $L$ and for the PPBC case. These distributions have been obtained by running a large number of samples ranging from 10 million of samples for $L=4$ down to 5 million for the largest size $L=9$. We have also inserted in the figure two vertical lines which indicate the limits for the range of values we have chosen for the fits of the scaling behavior of the finite-size excitation sector $(1-q \leqslant 0.07)$ and for the constant contribution corresponding to large scale excitations $(1-q \geqslant 0.25)$. We have chosen these values for the following reasons. First, as one can clearly see in Figs. 6 and 7 , the scaling region for small excitations survives up to excitation sizes $v \simeq L \times L$. This size provides a threshold value for the overlap $q_{\text {th }}$ below which the simple scaling $g(q) \simeq 1 /(1-q)^{\lambda_{l}+1}$ does not hold anymore,

$$
1-q_{\mathrm{th}}=1-\left(1-\frac{2 v}{V}\right) \simeq \frac{2 L^{2}}{R L^{2}} \simeq \frac{2}{R} .
$$

Second, there is a crossover region around $q \simeq q_{\text {th }}$. A careful look at Fig. 7 shows that the scaling region for small excitations ends around $1-q \simeq 0.07$. At this value, one observes a change of the slope of the curves just before entering the regime of large excitations where $g(q)$ becomes $q$ independent. For $1-q \geqslant 0.25$, the curves are rather constant and the result of a fit does not depend much on the choice $1-q$ $=0.25$. This second threshold value is indicated as the rightmost vertical bar in Fig. 7 .

In Fig. 8, we show the estimated values of effective lowest droplet exponent $\lambda_{l}^{\text {eff }}$ obtained in three different ways. The first estimate has been obtained by averaging the volume of all excitations for different lattice sizes as explained in Sec. III and then taking a logarithmic derivative, see Eq. (24). The second estimate has been obtained by considering the large excitation sector $(1-q \geqslant 0.25)$ and its $L, R$ dependence

$$
g(q) \simeq\left(R L^{2}\right)^{-\lambda_{l}}
$$

Averaging the excitation volume within this sector $(1-q$ $\geqslant 0.25$ ) and using again the corresponding logarithmic derivatives as in Eq. (24) yields the second estimate. The third estimate for $\lambda_{l}$ is obtained from a direct fit of $g(q)$ for small values of $1-q$ :

$$
g(q) \simeq(1-q)^{-1-\lambda_{l}}
$$




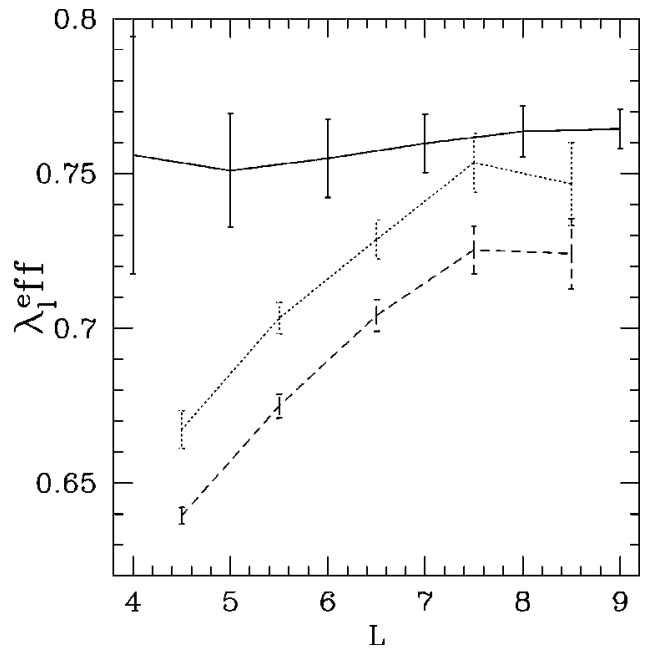

FIG. 8. Effective lowest droplet exponent $\lambda_{l}^{\text {eff }}$ versus $L$ for the PPBC case for $R=10$. We represent the values of $\lambda_{l}^{\text {eff }}$ obtained from fitting the distribution of $g(q)$ for small excitations (solid line), for large excitations (short dashed line), as well as the value obtained by fitting the average size of excitations (dotted line).

This third method is in fact the most direct one since it can be done for each size $L$ (while the other two estimates require a fit using data from two different lattice sizes $L$ and $\left.L^{\prime}\right)$. The first conclusion that we learn from Fig. 8 is that the ARA produces a great improvement on the estimated values of the exponent $\lambda_{l}$. The most stable measurement is the third estimate obtained by fitting the small-size spectrum of the excitations. In that case, $\lambda_{l}^{\text {eff }}$ is nearly constant with a value that converges to

$$
\lambda_{l}=0.77(1)
$$

in excellent agreement with the result (30) of the previous section.

Moreover, one also observes in Fig. 8 that the two other estimated values for $\lambda_{l}^{\text {eff }}$, obtained with the first and second methods, are strongly correlated. This shows that finitevolume corrections, which are expected to affect the value of the exponent obtained from the analysis of large-size excitations, do affect also the value of the exponent obtained by averaging over the whole spectrum. In addition, we also observe that the ARA for large $R$ strongly decreases the magnitude of finite-volume corrections. While on a square geometry, the effective exponent $\lambda_{l}^{\text {eff }}$ obtained from the average size of excitations took values in the range $0.52-0.62$ (see Fig. 2), with the ARA, we obtain for the same exponent values in the range $0.64-0.72$, which are much closer to the expected asymptotic value $0.77(1)$.

The same conclusion holds for the lowest droplet exponent $\theta_{l}$. In Fig. 9, we show the effective exponent $\theta_{l}^{\text {eff }}$ obtained by evaluating the logarithmic derivative as in Eq. (25). Note that finite-volume corrections are much smaller than with the squared lattices and as a result, the value of the effective exponent converges much faster to the expected value -2 . Using a fit of the form $\theta_{l}^{\text {eff }}(L)=\theta_{l}^{\text {eff }}(\infty)$ + const $/ L^{\alpha}$, one gets $\theta_{l}^{\text {eff }}(\infty)=-1.96(6)$ for the PPBC, the

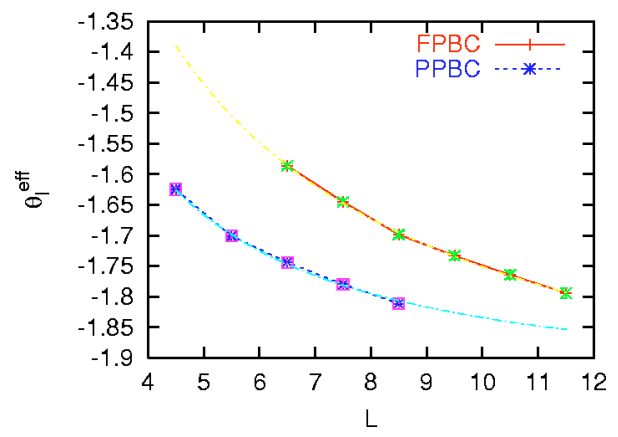

FIG. 9. Effective exponent $\theta_{l}^{\text {eff }}$ obtained via a logarithmic derivative for the $\mathrm{PPBC}$ and the FPBC. We also plot best fit curves which converge to $\theta_{l}^{\text {eff }}(L \rightarrow \infty)=-1.96(6)$ for the PPBC and to $\theta_{l}^{\text {eff }}(L \rightarrow \infty)=-2.12$ (11) for the FPBC.

best fit being also represented in Fig. 9. In this figure, we also show the same exponent obtained for the FPBC, where the best fit yields the asymptotic value $\theta_{l}^{\text {eff }}(\infty)$ $=-2.12(11)$. In both cases the fitting value we obtain for the exponent is $\alpha \simeq 1$. Note that the asymptotic values for $\theta_{l}^{\text {eff }}$ are well compatible with our prediction of Sec. II C, $\theta_{l}$ $=-2$ (see also the heuristic argument in Appendix A).

\section{FINITE-VOLUME CORRECTIONS (FVC) AND THEIR RELATION TO THE STATISTICS OF EXTREME VALUES}

What is the origin of these strong finite-volume corrections? Intuitively it is not difficult to find an explanation for the strong systematic finite-volume corrections in the lowest droplet exponent $\theta_{l}$. As the word lowest indicates, these exponents describe the statistical distribution of droplet excitations which are at the tail of the energy gap distribution that includes all possible high energy levels. As the volume of the system increases there is more available space to find excitations with lower energy gap. This implies that there is more probability to find a lowest droplet with an energy smaller than a given threshold value $E^{*}$ and therefore the average energy of the lowest droplet is expected to decrease with $L$. However, this simple fact does not give any intuitive indication of how the effective exponents $\theta_{l}^{\text {eff }}(L), \lambda_{l}^{\text {eff }}(L)$ systematically change with $L$.

To understand the origin of finite-volume corrections in the value of $\theta_{l}$ we have focused our attention on the behavior of the upper bound exponent $\theta_{l}^{1}$ describing the statistics of the lowest one-spin excitations as described in the Appendix A. The gap distribution corresponding to these excitations can be obtained from the local-field distribution evaluated at the ground state. We have numerically computed this distribution for different sizes, the results are shown in Fig. 10. As discussed in Appendix A, the local-field distribution has a finite weight at zero field and is a self-averaging quantity. As the local-field distribution is self-averaging, the probability distribution for the lowest one-spin excitations corresponds to the extreme value statistics of the local-field distribution $p_{1}(h)$ where $h$ stands for the local field which we assume to be positive as the gap is given by its absolute value (the subindex 1 is used to stress that this distribution describes 


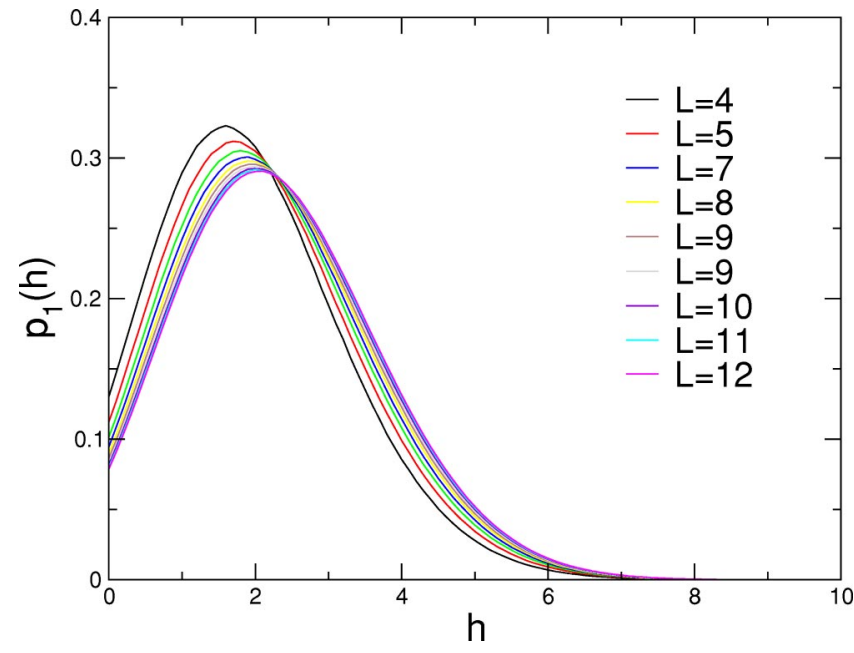

FIG. 10. Local-field distribution for different lattice sizes with FFBC boundary conditions.

energy gaps for one-spin excitations only). If $P_{1}(h)$ stands for the probability distribution of the smallest local fields, then $P_{1}(h)$ can be easily related to $p_{1}(h)$ by standard probability arguments (see for instance, Ref. 26). Although the argument is very general, here we apply it to one-spin excitations. For a given sample, the lowest value $h$ is selected as the minimum value among all the possible $V$ local fields $h_{i}$ at each lattice site. The probability $P_{1}(h)$ is given by the expression

$$
\begin{aligned}
P_{1}(h) & =V p_{1}(h)\left(1-\int_{h}^{\infty} p_{1}\left(h^{\prime}\right) d h^{\prime}\right)^{V-1} \\
& =-\frac{\partial}{\partial h}\left(\int_{h}^{\infty} p\left(h^{\prime}\right) d h^{\prime}\right)^{V}
\end{aligned}
$$

which accounts for all possible ways the value $h$ coincides with the minimum value obtained among all different $V$ local fields distributed according to the $p_{1}(h)$. The last identity shows that $P_{1}(h)$ is normalized. This probability can be explicitly worked out in the large $V$ limit

$$
P_{1}(h)=-\frac{\partial}{\partial h} \exp \left[-V g_{1}(h)\right]=V g_{1}(h) \exp \left[-V g_{1}(h)\right]
$$

Up to second order in $h$ the function $g_{1}(h)$ is given by

$$
g_{1}(h)=p_{1}(0) h+\frac{p_{1}^{\prime}(0)+\left[p_{1}(0)\right]^{2}}{2} h^{2}
$$

From Eq. (36) we immediately learn that the gap distribution is an exponential with a sub-leading Gaussian correction whose magnitude decreases as $1 / V$. Actually, plotting $P_{1}(h) / V$ as function of the scaling variable $x=h V$ one gets

$$
\frac{P_{1}(h)}{V}=g_{1}^{\prime}(x / V) \exp \left[-x p_{1}(0)-\frac{p_{1}^{\prime}(0)+\left[p_{1}(0)\right]^{2}}{2 V} x^{2}\right] .
$$

In the large $V$ limit $g_{1}^{\prime}(x / V) \rightarrow p_{1}(0)$ and the coefficient in front of the Gaussian correction goes asymptotically to zero, therefore the distribution $P_{1}(h)$ converges to an exponential as expected,

$$
P_{1}(h)=V p_{1}(0) \exp \left[-V p_{1}(0) h\right]
$$

in agreement with the scaling relation (13). We can now understand the deviations from the pure exponential behavior discussed in Sec. III in the context of the inset (a) shown in Fig. 3. They are simply consequences of the finite-volume corrections of the extreme values of the gap distribution for all energy levels (and not only one-spin excitations as we are discussing here). However, moving our discussion from the one-spin case to the absolute lowest excitation distribution $P(E)$, we do not have a clear physical insight about what the corresponding distribution $p(E)$ should be. In other words, while $p_{1}(E)$ is a self-averaging distribution with $P_{1}(E)$ being its extremal value distribution, we do not know how to construct a self-averaging $p(E)$ that yields the $P(E)$ we are numerically evaluating. Still, from the $P(E)$ 's shown in Fig. 3 , the parameters $p(0), p^{\prime}(0)$ that characterize such a $p(E)$ can be evaluated. To evaluate them, the best way is to analyze the cumulative distribution $\mathcal{P}(E)=\int_{E}^{\infty} d E^{\prime} P\left(E^{\prime}\right)$ which from Eq. (36) we can assume to be $\mathcal{P}(E)=\exp [-V g(E)]$. Thus we can fit $\mathcal{P}(E)$ with an exponential with Gaussian corrections $A \exp \left[-B x-C x^{2} / 2\right]$ whose fitting parameters are related to $p(0)$ and $p^{\prime}(0)$. The best fits yield the following values: $p(0) \approx 0.2$ and $p^{\prime}(0)=0.3$.

Coming back to our original goal we discuss now the finite-volume corrections for the estimate $\theta_{l}^{\text {eff }}$, as shown in Fig. 4. From the distribution (36) describing the whole spectrum of excitations we can express the effective exponent (25) for $L \gg 1$ as

$$
\theta_{l}^{\mathrm{eff}}(L)=\frac{\partial \ln [\bar{E}(L)]}{\partial \ln (L)}
$$

The computation of $\bar{E}(L)$ is quite straightforward as it is given by the simple relation

$$
\bar{E}(L)=\int_{0}^{\infty} E P(E) d E=\int_{0}^{\infty} d E \exp [-V g(E)],
$$

where we have used Eq. (36) plus an integration by parts. The integral, up to second order in $1 / V$ yields

$$
\bar{E}=\frac{1}{V p(0)}-\frac{p^{\prime}(0)+[p(0)]^{2}}{V^{2}[p(0)]^{3}}+O\left(\frac{1}{V^{3}}\right) .
$$

Inserting this result in Eq. (40) we finally get

$$
\theta_{l}^{\mathrm{eff}}(L)=-d+\frac{d}{V}\left(1+\frac{p^{\prime}(0)}{[p(0)]^{2}}\right)+O\left(\frac{1}{V^{3}}\right) .
$$

This shows that $\theta_{l}^{\text {eff }}(L)$ approaches $-d$ from below [as $p^{\prime}(0)$ is positive]. On the other hand the magnitude of the finite-volume corrections can be pretty large if $p^{\prime}(0) /[p(0)]^{2} \gg 1$. For instance, if one takes the results obtained from the analysis of one-spin excitations one gets 


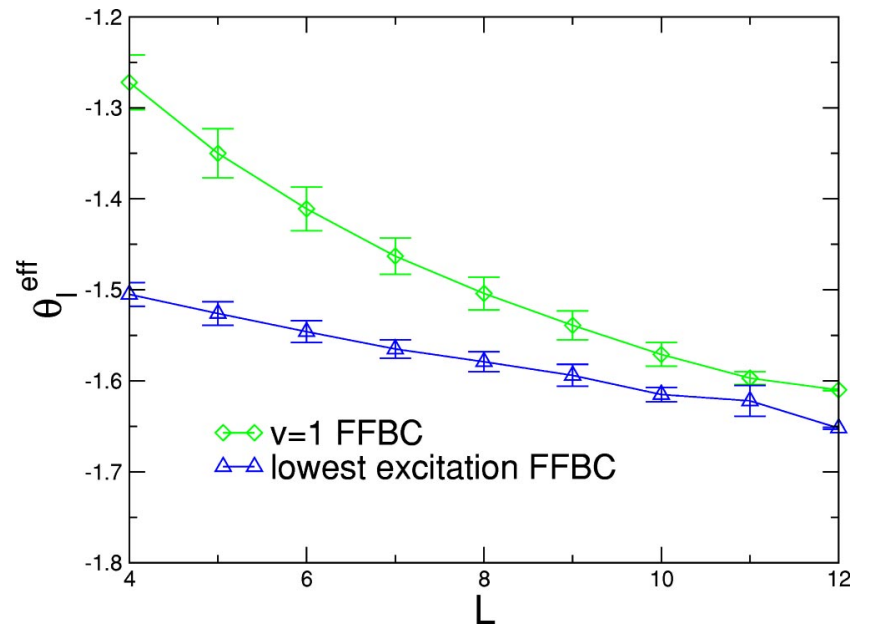

FIG. 11. Effective droplet exponent $\theta_{l}^{\text {eff }}$ versus $L$ for the FFBC case, computed using logarithmic derivatives (see text). We show the exponent obtained for one-spin excitations $(v=1)$ in comparison to the one obtained from the whole distribution of gaps.

$p_{1}(0) \simeq 0.069, p_{1}^{\prime}(0) \simeq 0.125$ yielding $p_{1}^{\prime}(0) /\left[p_{1}(0)\right]^{2} \simeq 27$ which is indeed large. Inserting these values in Eq. (43) we obtain an estimation for $\theta_{l}^{\text {eff }}(L=12)=-1.65$ in good agreement with numerical results (see Fig. 11).

If we insert the previous estimated values for the whole spectrum of excitations extracted from the $P(E)$ 's in Fig. 3, we obtain $p^{\prime}(0) /[p(0)]^{2} \simeq 7.5$. From Eq. (43) it follows that $\theta_{l}^{\text {eff }}(L) \simeq-2(1-7.5 / V)$, which for $L=11$ yields $\theta_{l}^{\text {eff }}$ $=-1.87$. All in all, the magnitude of the effective exponent $\theta_{l}$ is well compatible with the reported value $\theta_{l}^{\text {eff }}$ used in the inset (a) in Fig. 3 for the PP case. Note that the FVC corrections to $\theta_{l}^{\text {eff }}$ obtained from the local-field distributions in the FF case are much larger than FVC corrections in the PP case in agreement with ARA results (see Fig. 9). From this analysis it becomes clear that to significantly reduce the magnitude of the finite-volume corrections in the value of $\theta_{l}$ (let us say $\theta_{l} \simeq-1.95$ ), we would need larger volumes beyond $20 \times 20$.

\section{COMPACTNESS OF THE LOWEST ENERGY DROPLETS}

One intriguing question about the droplet excitations concerns their topological properties. Kawashima and $\mathrm{Aoki}^{13}$ have argued that droplet excitations are not compact. Instead, their volume has a fractal structure as the number of lattice points included in the droplet scales with its spanning length (which is a measure of the length scale of the droplet) with an exponent smaller [around 1.80(2)] than the dimension of the system (2).

To answer this question we have computed the surface, i.e., the perimeter $P$, of all lowest droplets. The relation between the average perimeter as function of the size $v$ of the excitation depends on both the fractal dimension of the surface or perimeter $d_{s}$ and the volume $d_{v}$ of the lowest droplets. $d_{s}$ and $d_{v}$ can be defined in terms of the spanning length $l$ of the droplet which can be defined in different ways. For example, one could use the gyration radius, the average distance between the sites contained in the cluster, or the maximum distance among the sites of the cluster. As the typical length scale of our lowest droplets is small, $l \simeq 10$, we have not attempted to estimate it as this can strongly depend on the precise definition of the spanning length. Here, we restrict ourselves to investigate the perimeter-volume dependence. In terms of the spanning length $l$ the surface fractal and volume fractal dimensions $d_{s}, d_{v}$ of the droplets are defined as

$$
\begin{aligned}
& l \sim P^{1 / d_{s}}, \\
& l \sim v^{1 / d_{v}}
\end{aligned}
$$

which combined give

$$
P \sim v^{d_{s} / d_{v}}
$$

In Fig. 12 we show $P(v)$ as a function of $v$ for different lattice sizes. As can be seen, FVC are important for large volumes. However, there is an enveloping curve that is independent of $L$ for small volumes and spans a progressively increase range of volumes as $L$ increases. This enveloping curve is excellently fitted (continuous curve) by the scaling relation (46) and yields an estimate

$$
\frac{d_{s}}{d_{v}} \simeq 0.632(2)
$$

consistent with the results reported by Kawashima and Aoki, $d_{s} / d_{v}=0.61(1)$, obtained with a completely different method.

\section{CONCLUSIONS}

We have shown that a proper description of lowtemperature properties in two-dimensional Gaussian spin glasses can be done in terms of the lowest droplet exponents (LDE's) $\lambda_{l}$ and $\theta_{l}$ describing the spectrum of lowest excitations. $\lambda_{l}$ describes the spectrum of sizes of the lowest energy droplets, while $\theta_{l}$ describes the typical energy cost of these lowest droplets whatever their size. Assuming that $\theta_{l}=-d$ one concludes that the LDE $\lambda_{l}$ fully characterizes the spinglass phase. Although independent numerical estimates of $\theta_{l}$ and $\lambda_{l}$ show strong finite-volume corrections, the thermal exponent $\theta=\theta_{l}+d \lambda_{l}$ can be well estimated giving the results $(29),(30)$

$$
\theta=-0.46(1), \quad \lambda_{l}=0.770(5),
$$

showing that $\theta<\theta_{\mathrm{DW}}=-0.287(4) .{ }^{11}$ Our estimates (48) have been confirmed via an aspect-ratio analysis which provides estimates much less influenced by finite-volume corrections. Moreover, the result $\theta_{l}=-2$ (that is believed to be correct for spin glasses with coupling distributions with finite weight at zero coupling, see the Appendix A) has been numerically confirmed by the aspect-ratio analysis. To sum up, McMillan's excitations are not the typical low-lying excitations and our approach offers a new and independent way to estimate the thermal exponent $\theta$ without the need to generate 


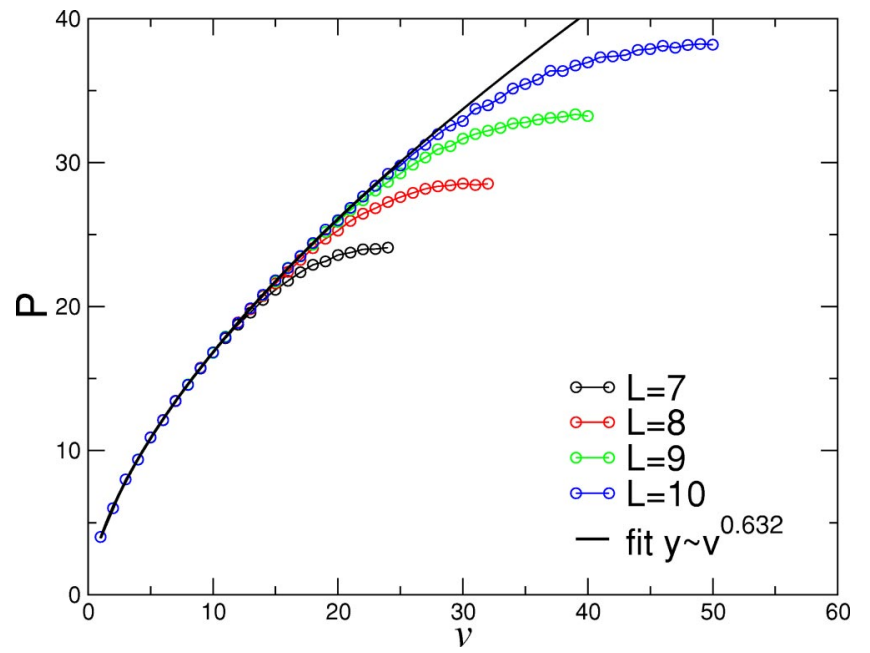

FIG. 12. Perimeter $(P)$ of the droplet versus its volume $(v)$. The solid line corresponds to the fit (46) with $d_{s} / d_{v}=0.632(2)$.

typical low-lying excitations by looking at the new ground state of the system after perturbing it.

We think that discrepancies on the value of the thermal exponent $\theta$ reported by comparing nonperturbative methods (such as finite-temperature transfer-matrix calculations and the present lowest droplet analysis) with perturbative methods such as domain-wall calculations (or perturbations induced by introducing a coupling term in the energy function that induces a large-scale excitation) are serious enough to be taken as a clear indication that our knowledge of the lowtemperature properties of the 2D GISG is still inadequate. In this direction we want also to recall the issue of multifractality and the possibility that different exponents could describe the zero-temperature critical point. Is this really possible? Well, to our knowledge no exact result precludes this possibility and, although purely speculative at the present stage, one should seriously think about it. Altogether, the present analysis suggests that the excitations in 2D GISG's are very different from the compact droplets proposed in the context of the droplet model. If this were true, the implications of the 2D studies in larger dimensions could be important. There are many routes that can be followed to understand better what is going on and the origin of this discrepancy. Certainly, with the outstanding accuracy of present algorithms to compute ground states in 2D, it would be very interesting to revisit the analysis of the statistics of the large scale excitations generated by imposing a uniform magnetic field. "Old" results by Rieger et al. ${ }^{8}$ give an estimate for $\theta$ that is compatible with our estimate rather than to the domain-wall estimate. This would be an independent check of our values, but using a perturbation method with an appropriate neutral observable such as the global magnetization as has been explained in Sec. I before Eq. (1).

The proposed method may appear venturesome as, to our present knowledge, there is no numerical study in the field along this line of research. However, as explained in Sec. I, recent studies on the disordered Anderson model ${ }^{20}$ have revealed that the analysis of the lowest excitation provides a good description of the localized phase. More studies are certainly required to understand better the reliability of the present method to investigate the critical properties of spin glasses. One disadvantage of our approach is that a huge number of samples is needed to reasonably sample largescale excitations. However, as we saw in Sec. V, the behavior of the $g(q)$ for small $1-q$ can be extracted with a modest number of samples. The advantage, as has been already stressed in Sec. I, is that we do not introduce any external perturbation to generate the excitations.

Finally, we want to comment on the extension of this approach to other models. Of course, the immediate extension one could think of is the $2 \mathrm{D} \pm J$ model. However, the analysis of this model appears quite troublesome. This model does not have a continuous gap distribution but a discrete one that introduces further complications. As the ground state is not unique, one has to redefine the full analysis to properly define the spectrum of lowest excitations. The discreteness of variables could have some unexpected effects in the present approach as seems to happen also with domainwall calculations. ${ }^{9,11}$ It is more natural to extend the research to other models such as $2 \mathrm{~d}$ ISG with other continuous coupling distributions without gap [e.g., characterized by $P(J)$ $\sim|J|^{\alpha}$ for $\left.|J| \rightarrow 0\right]$, Migdal-Kadanoff spin glasses (where both the ground state and the first excitation could be feasibly found with an appropriate algorithm), Gaussian spin glasses beyond $d=2$ (where unfortunately, algorithms are much less effective than in 2D as the finding of the ground state becomes a NP complete problem) and finally meanfield spin-glass models where the zero temperature exponents are known and maybe the spectrum of lowest excitations could be analytically tackled. Preliminary results in this case $^{27}$ confirm that the present analysis describes pretty well the data for rather small sizes. We are pretty confident that, in the near future, new results and evidence will finally resolve this interesting problem.

Note added after completion of this work: While this paper was submitted, Hartmann and Moore ${ }^{28}$ have reported some results in 2D Gaussian spin glasses where they generate large scale excitations by a perturbation technique where they fix some spins in the lattice and generate the smallest energy droplet. They show that the thermal exponent takes the value -0.47 for sizes $L \leqslant 20$ and crosses over to the value -0.29 for larger sizes. They interpret these data as evidence that -0.46 only holds in the small $L$ regime. However this interpretation needs to be taken with caution because of two following reasons. (1) Their data and our data cover different regimes: the typical energy of the droplets is much different in their approach than in our approach, their energies being at least one order of magnitude larger than ours, hence extrapolation of the finite-size effects they measure to our energy scale is not obvious at all. (2) Their data can be interpreted in the opposite way: for small sizes their excitations are indeed typical and scale properly with the exponent -0.46 , however, for larger sizes their droplets are not typical anymore since their perturbation does not properly select them (as we have reasoned along this paper), therefore they overestimate the energy of the typical ones giving a value of the thermal exponent $\theta$ compatible with the domain wall estimate. Which one is the correct explanation 
cannot be decided at present, however, we are confident that measurements for larger sizes using our method should resolve this issue.

\section{ACKNOWLEDGMENTS}

We are grateful to J.-P. Bouchaud, D. Huse, O. Martin, M. A. Moore, and A. P. Young for useful comments. We have been supported by the Spanish Ministerio de Ciencia y Tecnología, Project Nos. BFM2001-3525 (F.R.) and Grant No. AP98-36523875 (M.S.). M.P. and F.R. acknowledge support from the French-Spanish Collaboration (Picasso program and Acciones Integradas HF1998-0097). Funding from the European Science Foundation through the SPHINX program is also acknowledged.

\section{APPENDIX A: HEURISTIC PROOF OF THE IDENTITY $\theta_{l}=-d$}

In this appendix we show that $\theta_{l}=-d$. In what follows we do not attempt to present a rigorous proof but we content ourselves to present an heuristic argument. The argument has two parts: first we show that $-d$ is an upper bound, next we show that the upper bound is the exact value. For the upper bound the argument is well known and goes as follows. Consider the ground state and all possible one-spin excitations. Because one-spin excitations are not necessarily the absolute lowest ones, the statistics of the lowest one-spin excitations must yield an upper bound $\theta_{l}^{1}$ for the value of $\theta_{l}, \theta_{l} \leqslant \theta_{l}^{1}$. The statistics of the lowest one-spin excitations is determined by the behavior of the ground-state local field distribution $p(h)$ in the limit $h \rightarrow 0$. If $p(h)$ is self-averaging and $p(0)$ is finite (in the large-volume limit) then the statistics of the lowest excitations must be governed by the exponent $\theta_{l}^{1}=-d$. Although we do not know a precise mathematical proof of the statement that $p(0)$ is finite, it looks quite intuitive. $^{29}$ In any short-range system with a frustrated ground state and a coupling distribution with finite density at zero coupling, we may expect a finite probability to find a cage containing a spin coupled to its neighbors by a set of weak bonds which produce a vanishing net local-field acting on that spin. This argument should generally hold for $d$ $\geqslant 2$. Moreover, as its name indicates, the local-field distribution is a local observable. An argument in the manner of Brout proves that it should be self-averaging as all possible local field values are realized across the whole lattice (our numerical results in the 2D GISG confirm this conclusion, see Sec. VI). The next part of the argument consists in proving that an identical upper bound is valid by considering excitations with size strictly larger than 1 but finite. The upper bound derived for the one-spin excitations must necessarily hold for finite-size excitations beyond one-spin excitations (for instance, two spins, three spins, and so on) as the gap corresponding to the finite-size excitations can always be written as a linear combination of a finite number of local fields with coefficients which depend on the ground state configuration. It is easy to verify that the aforementioned properties of the local-field distribution $p(h)$ imply that the new gap distribution has a finite weight at zero gap and is self-averaging. This argument, however, cannot be extended to large-scale (with $v \sim V$ ) excitations in a straightforward way because the distribution for the corresponding gap distribution corresponds to an infinite sum of terms in the $V$ $\rightarrow \infty$ limit. However, once we argue that $\theta_{l}$ is an upper bound valid for all finite-size excitations it can be concluded that this upper bound must coincide with the exponent $\theta_{l}$ describing the probability of the absolute lowest excitations. From Eq. (4) the fraction of large scale excitations $v \rightarrow V$ is given by $V g_{V}=1 / V^{\lambda}$. In general $\lambda>0$ so this fraction vanishes (this fraction is finite only in $d=1$ where $\lambda=0$, but this case is trivial as the surface of large scale droplets in $d=1$ contains only a finite number of broken bonds) in the infinitevolume limit and finite-size excitations determine the result $\theta_{l}=-d$ as they dominate the spectrum of lowest excitations. Moreover, if large-scale excitations yield a different value for $\theta_{l}$ this would imply that boundary conditions could affect the value of the thermal exponent. That would be quite unusual as this would mean that the exponents of the $T=0$ fixed point would depend on the boundary conditions.

\section{APPENDIX B: TRANSFER MATRIX ALGORITHMS}

In this appendix, we will briefly explain how we determine the ground state and the first excited state. We will work on a square lattice of size $L \times L$. The energy associate to a configuration of spins $S(i, j)$ with a fixed configuration of disorder $J^{x}(i, j)$ and $J^{y}(i, j)$ is

$$
\begin{aligned}
E= & \sum_{i=1, L-1} \sum_{j=1, L} J^{x}(i, j) S(i, j) S(i+1, j) \\
& +\sum_{i=1, L} \sum_{j=1, L-1} J^{y}(i, j) S(i, j) S(i, j+1) \\
& +B_{1} \sum_{j=1, L} J^{x}(L, j) S(L, j) S(1, j) \\
& +B_{2} \sum_{i=1, L} J^{y}(i, L) S(i, L) S(i, 1),
\end{aligned}
$$

where $B_{1}$ and $B_{2}$ correspond to the choice of boundary conditions. Here we will consider three cases : Periodic-Periodic boundary conditions (PPBC's) with $B_{1}=B_{2}=1$, freeperiodic boundary conditions (FPBC's) with $B_{1}=1, B_{2}=0$ (or equivalently $B_{1}=0, B_{2}=1$ ), and free-free boundary conditions (FFBC's) $B_{1}=B_{2}=0$. We will only consider the case with a Gaussian distribution of the bond disorder $J^{x}, J^{y}$. To determine the ground state and the first excited states, we proceed as follows: we start by associating a weight for each configurations of spins in the first row of the lattice $S(1,1), S(1,2), \ldots, S(1, L)$ :

$$
\begin{aligned}
& W[S(1,1), S(1,2), \ldots, S(1, L)]=B_{2} J^{y}(1, L) S(1, L) S(1,1) \\
& \quad+\sum_{i=1, L-1} J^{y}(1, i) S(1, i) S(1, i+1) .
\end{aligned}
$$

Next, we start iterating the transfer matrix using a sparsematrix factorization. ${ }^{30}$ The first iteration gives 


$$
\begin{aligned}
W^{1}[ & S(1,2), \ldots, S(1, L), S(2,1)] \\
= & \max _{S(1,1)}\left\{J^{x}(1,1) S(1,1) S(2,1)\right. \\
& +W[S(1,1), \ldots, S(1, L)]\} .
\end{aligned}
$$

Since we are also interested in the first excited state, we define the second largest weight

$$
\begin{aligned}
W^{2}[ & S(1,2), \ldots, S(1, L), S(2,1)] \\
= & \min _{S(1,1)}\left\{J^{x}(1,1) S(1,1) S(2,1)\right. \\
& +W[S(1,1), \ldots, S(1, L)]\} .
\end{aligned}
$$

In the following, we will use the simplified notation

$$
\begin{aligned}
W(i, j) \equiv & W[S(i, j), \ldots, S(i, L), S(i+1,1), \ldots, S(i+1, j \\
& -1)] .
\end{aligned}
$$

Thus Eqs. (B3),(B4) become

$$
W^{1}(1,2)=\max _{S(1,1)}\left[J^{x}(1,1) S(1,1) S(2,1)+W(1,1)\right]
$$

and

$$
W^{2}(1,2)=\min _{S(1,1)}\left[J^{x}(1,1) S(1,1) S(2,1)+W(1,1)\right] .
$$

At the next iteration, the numbers of possible weight will again be multiplied by two but we will keep only the two largest ones defined as

$$
\begin{aligned}
W^{1}(1,3)= & \max _{S(1,2)}\left[J^{x}(1,2) S(1,2) S(2,2)\right. \\
& \left.+J^{y}(2,1) S(2,1) S(2,2)+W^{1}(1,2)\right] \\
W^{2}(1,3)= & \max \left\{\operatorname { m i n } _ { S ( 1 , 2 ) } \left[J^{x}(1,2) S(1,2) S(2,2)\right.\right. \\
& \left.+J^{y}(2,1) S(2,1) S(2,2)+W^{1}(1,2)\right], \\
& \max _{S(1,2)}\left[J^{x}(1,2) S(1,2) S(2,2)\right. \\
& \left.\left.+J^{y}(2,1) S(2,1) S(2,2)+W^{2}(1,2)\right]\right\} .
\end{aligned}
$$

The general iteration relations are

$$
\begin{aligned}
W^{1}(i, j)= & \max _{S(i, j-1)}\left[J^{x}(i, j-1) S(i, j-1) S(i+1, j-1)\right. \\
& +J^{y}(i+1, j-2) S(i+1, j-2) S(i+1, j-1) \\
& \left.+W^{1}(i, j-1)\right], \\
W^{2}(i, j)= & \max \left\{\operatorname { m i n } _ { S ( i , j - 1 ) } \left[J^{x}(i, j-1) S(i, j-1) S(i+1, j-1)\right.\right. \\
+ & J^{y}(i+1, j-2) S(i+1, j-2) S(i+1, j-1) \\
+ & \left.W^{1}(i, j-1)\right], \\
& \max _{S(i, j-1)}\left[J^{x}(i, j-1) S(i, j-1) S(i+1, j-1)\right. \\
+ & J^{y}(i+1, j-2) S(i+1, j-2) S(i+1, j-1) \\
+ & \left.\left.W^{2}(i, j-1)\right]\right\} .
\end{aligned}
$$

In addition, each time that we end the construction of a new row, we must add the boundary term

$$
\begin{aligned}
W^{1}(i, 1) & \rightarrow W^{1}(i, 1)+B_{2} J^{y}(i, L) S(i, L) S(i, 1) ; W^{2}(i, 1) \\
& \rightarrow W^{2}(i, 1)+B_{2} J^{y}(i, L) S(i, L) S(i, 1) .
\end{aligned}
$$

We still have to take in account the boundary condition corresponding to $B_{1}$. The two types of boundary conditions (free and periodic) have to be considered separately.

Free boundary condition $B_{1}=0$. In that case, we iterate up to the construction of the weights associated with the configurations of the spins $S(L, 1), \ldots, S(L, L)$. The energy of the ground state $\left(E_{0}\right)$ is then simply the maximum among all the weights $W^{1}(L, 1)$ :

$$
E_{0}=\max _{\{S(L, 1), \ldots, S(L, L)\}}\left[W^{1}(L, 1)\right] .
$$

We call $\left\{S^{0}(L, 1), \ldots, S^{0}(L, L)\right\}$ the configuration of spins on the last row for the ground state. The energy of the first excited state is the second largest weight among $W^{1}(L, 1)$ and $W^{2}(L, 1)$ :

$$
\begin{aligned}
E_{1}= & \max \left\{\max _{\{S(L, 1), \ldots, S(L, L)\} \neq\left\{S^{0}(L, 1), \ldots, S^{0}(L, L)\right\}}\right. \\
& \left.\times\left[W^{1}(L, 1)\right], \max _{\{S(L, 1), \ldots, S(L, L)\}}\left[W^{2}(L, 1)\right]\right\} .
\end{aligned}
$$

Periodic boundary case $B_{1}=1$. We first choose one configuration of spins on the first row $S^{i}(1,1), S^{i}(1,2), \ldots, S^{i}(1, L)$. The weight of this configuration is defined as in Eq. (B2). The weight of all the other configurations of spins on this first row are fixed to an arbitrary large negative number. Next we iterate the transfer matrix as described above, up to the construction of the weights $W^{1}(L, 1)$ and $W^{2}(L, 1)$. Finally, we iterate one additional row, with bonds $J^{x}(L, i)$ and $J^{y}(L+1, i)=0$. Next, we store the two weights $W^{1}$ and $W^{2}$ associated to the initial spins configuration $S^{i}(1,1), S^{i}(1,2), \ldots, S^{i}(1, L)$. We denote these two weights by

$$
\begin{aligned}
\mathcal{W}^{1} & {\left[S^{i}(1,1), S^{i}(1,2), \ldots, S^{i}(1, L)\right] } \\
& \equiv W^{1}\left[S(L+1,1)=S^{i}(1,1), S(L+1,2)\right. \\
& \left.=S^{i}(1,2), \ldots, S(L+1, L)=S^{i}(1, L)\right] \\
\mathcal{W}^{2} & {\left[S^{i}(1,1), S^{i}(1,2), \ldots, S^{i}(1, L)\right] } \\
& \equiv W^{2}\left[S(L+1,1)=S^{i}(1,1), S(L+1,2)\right. \\
& \left.=S^{i}(1,2), \ldots, S(L+1, L)=S^{i}(1, L)\right] .
\end{aligned}
$$

The energy of the ground state is the maximum on all the $\mathcal{W}^{1}$ :

$$
\begin{aligned}
E_{0}= & \max _{\left\{S^{i}(1,1), \ldots, S^{i}(1, L)\right.} \\
& \times\left\{\mathcal{W}^{1}\left[S^{i}(1,1), S^{i}(1,2), \ldots, S^{i}(1, L)\right]\right\},
\end{aligned}
$$

and we denote by $S^{0}(1,1), \ldots, S^{0}(1, L)$ the configuration of spins on the first row for the ground state. The energy of the first excited state is the second largest weight among $\mathcal{W}^{1}(L, 1)$ and $\mathcal{W}^{2}(L, 1)$ : 


$$
\begin{aligned}
E_{1}= & \max \left[\max _{\left\{S^{i}(1,1), \ldots, S^{i}(1, L)\right\} \neq\left\{S^{0}(1,1), \ldots, S^{0}(1, L)\right\}}\right. \\
& \times\left\{\mathcal{W}^{1}\left[S^{i}(1,1), S^{i}(1,2), \ldots, S^{i}(1, L)\right]\right\}, \\
& \max _{\left\{S^{i}(1,1), \ldots, S^{i}(1, L)\right\}} \\
& \left.\times\left\{\mathcal{W}^{2}\left[S^{i}(1,1), S^{i}(1,2), \ldots, S^{i}(1, L)\right]\right\}\right] .
\end{aligned}
$$

The construction of the ground state and of the first excited state is much more costly in computing time for the periodic case, since we have to repeat $2^{L}$ times the iterations, one time for each configuration $S^{i}(1,1), S^{i}(1,2), \ldots, S^{i}(1, L)$.

So far, we have only described how to compute the value of the energies associated with the ground state and the first excited state. Since we also want to determine the spins configurations for these two states, we have to store, at each iteration of the transfer matrix, the value of the spin on which one sums, as well as the value of the previous spins. Thus, for each $2^{L}$ weights $W^{1}(i, j-1)$, we have to store the configuration

$$
\begin{aligned}
& \mathcal{C}_{S(i, j-1), \ldots, S(i+1, j-2)} \\
& \quad \equiv[S(1,1), \ldots, S(1, L), S(2,1), \ldots, S(i, j-2)] .
\end{aligned}
$$

At the next iteration, we will build the weight $W^{1}(i, j)$ with the corresponding configuration

$$
\mathcal{C}_{S(i, j), \ldots, S(i+1, j-1)}=\left[\mathcal{C}_{S(i, j-1), \ldots, S(i+1, j-2)}, S(i, j-1)\right]
$$

with $S(i, j-1)$ the value of the spin which corresponds to the maximum in Eq. (B10). From this construction, we have access to the spins configurations of the ground state and the first excited state.

Finally, we should also add that this construction can be easily extended to the second excited state, etc. After Eq. (B9), we can easily define a third weight which would be associated to the second excited state, and so on.
${ }^{1}$ Spin Glasses and Random Fields, edited by A. P. Young (World Scientific, Singapore, 1998).

${ }^{2}$ M. Mézard, G. Parisi, and M. A. Virasoro, Spin Glass Theory and Beyond (World Scientific, Singapore, 1987).

${ }^{3}$ E. Marinari, G. Parisi, F. Ricci-Tersenghi, J. J. Ruiz-Lorenzo, and F. Zuliani, J. Stat. Phys. 98, 973 (2000); C. M. Newman and D. S. Stein, cond-mat/0105282 (unpublished).

${ }^{4}$ W. L. McMillan, J. Phys. C 17, 3179 (1984); A. J. Bray and M. A. Moore, ibid. 17, L463 (1984).

${ }^{5}$ A. J. Bray and M.A. Moore, in Heidelberg Colloquium on Glassy Dynamics and Optimization, edited by L. van Hemmen and I. Morgenstern (Springer-Verlag, Heidelberg, 1986); D. S. Fisher and D. A. Huse, Phys. Rev. B 38, 373 (1988); 38, 386 (1988).

${ }^{6}$ W. L. McMillan, Phys. Rev. B 29, 4026 (1984); 30, 476 (1984); 31, 342 (1985).

${ }^{7}$ A. J. Bray and M. A. Moore, Phys. Rev. B 31, 631 (1985); M. Palassini and A. P. Young, ibid. 60, R9919 (1999); A. K. Hartmann, Phys. Rev. E 59, 84 (1999).

${ }^{8}$ H. Rieger, L. Santen, U. Blassum, M. Diehl, and M. Jünger, J. Phys. A 29, 3939 (1996).

${ }^{9}$ A. K. Hartmann and A. P. Young, Phys. Rev. B 64, 180404 (2002).

${ }^{10}$ A. C. Carter, A. J. Bray, and M. A. Moore, Phys. Rev. Lett. 88, 077201 (2002).

${ }^{11}$ A. K. Hartmann, A. J. Bray, A. C. Carter, M. A. Moore, and A. P. Young, Phys. Rev. B 66, 224401 (2002).

${ }^{12}$ N. Kawashima, H. Hatano, and M. Suzuki, J. Phys. A 25, 4985 (1992).

${ }^{13}$ N. Kawashima and T. Aoki, J. Phys. Soc. Jpn. 69, 169 (2000).

${ }^{14}$ S. Liang, Phys. Rev. Lett. 69, 2145 (1992); M. Ney-Nifle and A. P. Young, J. Phys. A 30, 5311 (1997).
${ }^{15}$ N. Kawashima and M. Suzuki, J. Phys. A 25, 1055 (1992); N. Kawashima, J. Phys. Soc. Jpn. 69, 987 (2000).

${ }^{16}$ J. Lamarcq, J.-P. Bouchaud, O. C. Martin, and M. Mézard, Europhys. Lett. 58, 321 (2002).

${ }^{17}$ A. K. Hartmann and A. P. Young, Phys. Rev. B 66, 094419 (2002).

${ }^{18}$ M. Picco, F. Ritort, and M. Sales, cond-mat/0106554 (unpublished).

${ }^{19}$ F. Ritort and M. Sales, J. Phys. A 33, 6505 (2000). 34, L333 (2001).

${ }^{20}$ Y. Gefen, R. Berkovits, I. V. Lerner, and B. L. Altshuler, Phys. Rev. B 65, 081106(R) (2002); R. Berkovits, Y. Gefen, I. V. Lerner, and B. L. Altshuler (unpublished).

${ }^{21}$ M. Picco, F. Ritort, and M. Sales, cond-mat/0106592 (unpublished).

${ }^{22}$ M. Palassini and A. P. Young, Phys. Rev. Lett. 85, 3017 (2000); F. Krzakala and O. Martin, ibid. 85, 3013 (2000).

${ }^{23}$ F. Krzakala and O. C. Martin, Phys. Rev. Lett. 85, 3013 (2000); J. Houdayer, F. Krzakala, and O. C. Martin, Eur. Phys. J. B 18, 467 (2000); A. K. Hartmann, Phys. Rev. E 63, 016106 (2001).

${ }^{24}$ This estimate was suggested to us by A. P. Young.

${ }^{25}$ M. A. Moore, cond-mat/0203469 (unpublished).

${ }^{26}$ M. Mézard and J. P. Bouchaud, J. Phys. A 30, 7997 (1997).

${ }^{27}$ M. Sales and F. Ritort (unpublished).

${ }^{28}$ A. K. Hartmann and M. A. Moore, Phys. Rev. Lett 90, 127201 (2003).

${ }^{29}$ We are indebted to D. Huse, who pointed out this argument as well as the validity of the general result $\theta_{l}=-d$.

${ }^{30}$ M. P. Nightingale, in Finite Size Scaling and Numerical Simulations of Statistical Systems, edited by V. Privman (World Scientific, Singapore, 1990). 